\title{
Modelling Damping Sources in Monopile-supported Offshore Wind Turbines
}

C. Chen, P. Duffour ${ }^{1}$

Department of Civil, Environmental and Geomatic Engineering, University College London, United Kingdom

${ }^{1}$ Corresponding author: p.duffour@ucl.ac.uk 


\title{
Modelling Damping Sources in Monopile-supported Offshore Wind Turbines
}

\begin{abstract}
Vibration damping in offshore wind turbines is a key parameter to predict reliably the dynamic response and fatigue life of these systems. Damping in an OWT originates from different sources, mainly, aerodynamic, structural, hydrodynamic and soil dampings. The difficulties in identifying the individual contribution from each damping source has led to considerable uncertainty and variation in the values recommended. This paper proposes simplified but direct modelling approaches to quantify the different damping contributions from the aerodynamic, hydrodynamic and soil interactions. Results from these models were systemically compared to published values and when appropriate with simulation results from the software package FAST. The range of values obtained for aerodynamic damping confirmed those available in the literature and Blade Element Modelling theory was shown to provide good results relatively efficiently. The influence of couplings between fore-aft and side-side directions on the aerodynamic damping contribution was highlighted. The modelling of hydrodynamic damping showed that this damping is much smaller than usually recommended and could be safely ignored for OWTs. Soil damping strongly depends on the soil specific nonlinear behaviour.
\end{abstract}

\section{Introduction}

Offshore wind farms (OWFs) are set to play a key role in achieving renewable energy targets around the world over the next decades. Many countries are making plans to develop offshore wind generation as the wind resource is stronger and steadier at sea [1]. As wind and waves are highly fluctuating loads and offshore wind turbines (OWTs) are slender structures, their dynamics must be considered carefully. Damping is critical in correctly predicting the dynamic behaviour of an OWT because it is the only factor that limits the amplitude of the response at resonance. The dynamic response in turn affects the severity of fatigue damage. Therefore, it is essential to quantify properly the damping from different sources to assess the fatigue damage of an OWT. There are five 
main sources of damping in OWTs: aerodynamic, hydrodynamic, structural, soil and supplemental damping provided by mechanical dissipating devices [2]. As OWTs are lightly damped structures, it is usual to assume that the total damping factor in the system is simply the sum of the damping factors from different sources as expressed by Equation (1):

$$
\zeta_{\text {Total }}=\zeta_{\text {Aero }}+\zeta_{\text {Struc }}+\zeta_{\text {Hydro }}+\zeta_{\text {Soil }}+\zeta_{\text {Damper }}
$$

where $\zeta_{\text {Total }}, \zeta_{\text {Aero }}, \zeta_{\text {Struc }}, \zeta_{\text {Hydro }}, \zeta_{\text {Soil }}, \zeta_{\text {Damper }}$ are respectively the total, aerodynamic, structural, hydrodynamic, soil and supplemental damping factors as a percentage of critical damping. The aerodynamic damping is the largest and most variable contributor so the other damping sources are sometimes lumped together under the term "additional damping". We shall call this the baseline damping in this paper to avoid any confusion between additional and supplemental. For consistency and ease of comparison, damping ratios are used throughout this paper (as opposed to logarithmic decrement) and all published damping values cited have been converted accordingly. The conversion of logarithmic decrement values into damping ratios (a division by $2 \pi$ ) as well the varying precision with which results are available in the literature has caused some difficulty in reporting damping values with a consistent level of significant figures. It should also be noted that the repeatability of damping measurements is usually quite poor (typically with $10-20 \%$ of variability) rendering the precision of many damping values reported here somewhat spurious. However, this was deemed necessary for the sake of comparison.

Damping in offshore and onshore wind turbines has been researched fairly extensively however the variations in published values for most damping sources remain large and this directly translates into uncertainty in the prediction of the dynamic response and fatigue life of the system. As a result, designs may be unsafe or overly conservative. Therefore, there is a need to better characterise each source of damping in OWT systems.

This paper initially started as a literature review on damping in OWTs intending to provide the offshore wind community with the most up-to-date damping values. This still constitutes the first part of this paper. However due to the lack of consensus for many sources of damping found in the literature, it was decided to complement the literature review with direct estimations of the various damping contributions through simple but physics-based models. This is the second part of the paper. The aims of this second part are first to better characterise the values reported but also to test the extent 
to which these simple models can reliably provide damping values. This may make the paper somewhat unusual but we believe that the two parts complement each other usefully.

For the second part of the paper, a finite element (FE) model was developed and used in conjunction with the coupled Aerodynamic Modelling Package FAST provided by NREL [3]. As most OWTs installed so far are supported on monopiles, this study focuses on this type of support structure [4].

Section 2 provides the comprehensive literature review highlighting areas of agreement as well the scatter in the published damping data. Section 3 presents the model and the general methodology used to estimate the damping. Sections 4, 5 and 6 describe the modelling approaches and results for the aerodynamics, hydrodynamic and soil damping respectively. The paper concludes in Section 7.

\section{Literature review}

Various physical processes contribute to the damping of OWTs and isolating them is not straightforward. Published analytical studies tend to concentrate on a particular damping source either by direct modelling or through back-calculation, i.e. estimating one source by subtracting other contributions to the overall damping. By contrast, experimental studies tend to focus on the overall damping in the system either in parked or operational regime, as this can be measured more readily. As a rough guide, GL recommend using a total damping ratio of $1 \%$ for parked turbines and $7 \%$ in operation [5].

\subsection{Experimental measurements of damping in parked OWTs}

Two main methods have been used to measure damping experimentally in OWTs depending on the type of excitation. Modal properties (including damping) have been obtained by Operational Modal Analysis (OMA) when the system is excited by ambient sources (wind and waves) or standard time- or frequency- domain damping identification techniques when the dynamic response of the system is due to a controlled measurable excitation. Applying a controlled excitation to an actual OWT presents a number of practical difficulties due to access and size. Loads from the environment could also influence the results. The so-called "rotor stop" test is the most commonly used technique (e.g. [6][7][8][9][10]) and consists in suddenly turning the blades into feathered 
position so that the tower experiences a downward step in rotor thrust. "Overspeed test" is a similar technique that has also been used extensively (e.g. [11][12][13][14][15]). Other types of excitations have also been used - for instance, Koukoura et al. used boat impact at sea level [16]. These techniques intrinsically reduce the influence of aerodynamic damping by keeping the rotor speed low or at zero, so that they are better suited at estimating the baseline damping. As a result, the difference in damping between the fore-aft (FA) and side-side (SS) directions tends to be small for idle turbines.

Using these various techniques, a range of values have been reported. Tarp-Johansen $e t$ al. [10] measured the basline damping ratio in a 3.5MW OWT using rotor stop tests and estimated it at $1.91 \%$. Versteijlen et al. [6] measured the baseline damping in 3.6MW Siemens OWTs using spectra of the bending moment at the base of the tower. They obtained a damping ratio of $3 \%$ for the first bending mode of the turbine. Damgaard et al. ([7][8][9]) used "rotor stop" tests and OMA on turbines subjected to ambient excitation in four wind farms. The rotor stop tests gave a first FA mode damping ratio in the range $2.39-2.55 \%$. Results from ambient excitation tests gave fairly close results with damping factors in the range $2.55-2.86 \%$. A series of comprehensive damping studies on 3MW Vestas V90 OWTs in Belwind Wind farm were completed by Shirzadeh et al. ([11][12][13][14][15]). Using overspeed tests and OMA under ambient excitation they measured the damping ratios of the first FA and SS modes at $1.05 \%$ and $1.27 \%$ respectively (with an installed tuned-mass damper kept inactive). Koukoura et al. [16] studied the damping in both parked and operating turbines excited by a boat impact and ambient excitation. They measured the FA and SS damping ratios of $1.8 \%$ and $1.9 \%$ respectively for a parked turbine. Dampers were installed and active in their study but their contribution was not specified.

In conclusion the damping ratios for parked turbines have been found to vary between $1 \%$ and $3 \%$ with reasonable agreement between published results.

\subsection{Experimental measurements of damping in operating OWTs}

Hansen et al. [17] used two experimental methods to estimate the aerodynamic damping in operating OWTs however no measurement of the baseline damping was provided in their study so their results are effectively overall damping values. One method estimated the damping from the decay in the OWT free response obtained after an artificial periodic pitching of the blades was stopped. The measured overall damping ratios ob- 
tained this way were extremely scattered, averaging around $\zeta^{F A} \sim 8 \%$ in the FA direction and $\zeta^{S S} \sim 2.4 \%$ in the SS direction. The other method used was OMA with wind excitation which gave an overall damping ratio of $13.2 \%$ for the FA mode and $8.0 \%$ in the SS mode. The difference between the results obtained from the two methods is large and OMA gave much higher values than those usually found in the literature. Koukoura et al. [16] found that the overall FA damping ratio for an operating OWT in a relatively high wind speed of $19 \mathrm{~m} / \mathrm{s}$ is $10.35 \%$ and $4.77 \%$ in the SS direction. However, this again seems to include some unspecified supplemental damping. In conclusion, there is a lot of uncertainty on the measured overall damping in operating wind turbines and it transpires that further work is needed to establish a reliable methodology to measure it.

\subsection{Aerodynamic damping}

Aerodynamic damping is mostly generated by the air drag experienced by the rotating blades oscillating in the airflow due to tower bending vibration [18]. The vibrating tower also experiences some air drag even if the rotor is idle but to a much lesser extent. Aerodynamic damping values have been reported in the range $4 \%$ to $8 \%$ in the FA direction (after rounding off). This dissipative effect is much less effective at damping the SS motion. In this case published damping factors range from $0.08 \%$ to $1.43 \%$.

Aerodynamic damping comes in such a wide range because it is strongly influenced by the wind speed, the rotation speed and the control system which changes the pitch angle of blades and the yaw angle of the rotor to optimise power output ([19][20]). A few papers have proposed theoretical derivations for the aerodynamic damping in OWTs. Salzmann and Tempel [21] summarised several analytical solutions for this damping in constant-speed turbines and they proposed their own analytical model for variablespeed turbines. Using Blade Element Momentum (BEM) theory [18] they obtained closed-form expressions that account for the relationship between wind speed and the motion of turbine. Comparing their numerical results to published experimental data they found that simulation largely underestimates the aerodynamic damping when the wind speed is much higher than the rated wind speed. Valamanesh and Myers [19] proposed a semi-analytical solution also based on BEM theory that predicts the aerodynamic damping for both fore-aft and side-side vibration. Their numerical results agree fairly well with results from FAST simulations. Tarp-Joahnsen et al [10] simulated the SS aerodynamic damping in a 3.5MW OWT using HAWCStab and found it to range from $0.08 \%$ to $1.43 \%$. Schafhirt and Muskulus [22] proposed a decoupled 
modelling process to model the dynamic behaviour of wind turbine in separate stages. Their approach includes a simplified derivation of the aerodynamic forces but their damping coefficients were eventually obtained by matching the response to that of a fully-coupled model.

Aerodynamic damping was studied experimentally by Ozbek and Rixen [23] on a 2.5 MW onshore turbine. The damping ratios were estimated using OMA from photogrammetry and laser vibrometry measurements. As onshore foundations are stiffer, onshore wind turbines are expected to experience much less soil damping and no hydrodynamic damping at all. Also they have no transition piece as such so their structural damping is bound to be low. Therefore, the baseline damping is likely to be very small so that a good estimate of the aerodynamic damping can be obtained by measuring the overall damping in operation. They found 5\% in the FA direction and around 0.5\% in SS mode. These values were in good agreement with results from matching HAWCS simulations they carried out. Although no other study has measured the aerodynamic damping directly, the overall damping values provided in the previous section can be used for this assuming standard values for the additional damping. Assuming $2 \%$ baseline damping component (our estimate), overall damping results from Hansen et al. [17] give an estimated $\zeta_{\text {Aero }}^{F A} \sim 6 \%$ and $\zeta_{\text {Aero }}^{S S} \sim 0.4 \%$ for the aerodynamic contributions using the controlled excitation. This is in line with other studies. Subtracting $2 \%$ from their OMA overall damping ratios gives $\zeta_{\text {Aero }}^{F A} \sim 11.2 \%$ and $\zeta_{\text {Aero }}^{S S} \sim 6 \%$. As Koukoura et al. [16] did not specify their supplemental damping, it seems prudent not to use their results in this way.

Therefore, published values concur that the aerodynamic damping is the strongest contribution in operation however both predicted or measured values can vary widely. Also there is currently not much published data for larger OWTs.

\subsection{Hydrodynamic damping}

Hydrodynamic damping comes from two sources: (1) wave radiation damping and (2) viscous damping due to hydrodynamic drag [24]. The wave radiation is proportional to wave velocities whereas viscous damping is proportional to the relative velocities squared [11]. Few studies have been conducted on hydrodynamic damping of OWTs. GL [5] suggests the values of $0.15 \%$ for viscous damping and $0.11 \%$ is proposed for the radiation damping in [25]. A radiation damping ratio of $0.24 \%$ was suggested by Tarp-Johansen et al. [10] after simulating the radiation/diffraction component using the 
wave modelling program WAMIT. Using HAWC2 time-domain simulations (implementing Morison's equation), Shirzadeh et al. [11] estimated the viscous hydrodynamic damping ratio at around $0.004 \%$. Again the reported values for this source of damping vary widely but for an OWT on monopile located in relatively shallow water, these damping values are consistently very low compared to other sources.

\subsection{Soil/foundation damping}

Soil damping plays a more prominent role when the turbine is idle or when the sideside behaviour is considered, as the aerodynamic damping is much smaller in these cases. Damgaard [9] modelled the influence of soil damping directly by introducing hysteretic springs in a cantilever model of the turbine to account for the Soil-Structure Interaction (SSI). From this they found the contribution of soil damping to range between $0.8 \%$ and $1.3 \%$ which is in good agreement with an experimental estimate of soil damping (1\%) they obtained by back calculation. Carswell et al. [26] used a similar method for converting hysteretic energy loss into a viscous rotational dashpot at mudline to represent OWT foundation damping in a lumped-parameter model. From numerical simulations of NREL 5MW reference turbine they found the soil damping ratio to be in the range of $0.17 \%-0.28 \%$ when inferred from free vibration results. However, a higher damping value of $0.72 \%$ was obtained from time history analyses in response to extreme wind and wave conditions. It should be noted that back calculating soil damping this way tends to increase the final uncertainty by propagating the uncertainties from other damping sources. Modelling the soil as a viscoelastic soil block in Abaqus, Tarp-Johansen et al. [10] found that a soil damping ratio of $0.56 \%$. They suggested that $0.80 \%$ should be used to account for the nonlinear behaviour of the soil. Their values are currently recommended by GL guidelines [5]. Although very few studies have attempted to model directly soil damping in OWT, the damping in a standard single pile in horizontal vibration has been the subject of many past papers (e.g. [27][28][29]). These could form the basis for modelling soil dissipation in monopiles. Geotechnical earthquake engineers also have a long history of studying soil damping (e.g. [30][31]). 


\subsection{Structural Damping}

Structural damping in offshore wind turbines has received very little specific attention. It is usually assumed to follow the behaviour of standard steel structures for which damping values are available. For instance, Eurocode 1 [32] recommends a value of $0.19 \%$ for unlined welded steel stacks without external thermal insulation. More specific values can be found in the Offshore Oil and Gas literature [33]. Structural damping in onshore wind turbines was measured by Schaumann and Seidel [34] and it was found to be between $0.2 \%$ to $0.5 \%$ (excluding soil damping). Ozbek and Rixen [23] measured it at $0.3 \%$ in the FA direction and between $0.3 \%$ and $0.9 \%$ in the SS direction. However structural damping in OWTs could be larger (from $0.5 \%$ to $1.5 \%$ according to [11]) as the damping from other structural parts like the grouted connection may have a significant contribution. Offshore wind farms also tend to have much larger turbines than those investigated in the studies reported in this section so it is not clear how reliably their measurements can be generalised to current larger OWTs.

\subsection{Summary}

The damping estimations from past papers are summarised in Table 1 for comparison. These values reflect the range and uncertainties in damping from different sources for different directions and operational regimes. Cells with a blue background represent values on which many papers agreed or when the range of proposed values is quite narrow thus indicating a higher degree of confidence than the yellow cells for which these conditions are not met.

Table 1 Damping ratios range based on published results. Yellow cells indicate large uncertainties while blue cells indicate smaller uncertainties.

\begin{tabular}{|l|c|l|l|l|l|l|}
\hline $\begin{array}{l}\text { Operation } \\
\text { Condition }\end{array}$ & $\begin{array}{l}\text { Direc- } \\
\text { tion } \\
\text { damping }\end{array}$ & $\begin{array}{l}\text { Overall } \\
\text { damping } \\
\text { damping }\end{array}$ & $\begin{array}{l}\text { Structural } \\
\text { Aerodynamic }\end{array}$ & $\begin{array}{l}\text { Soil damping } \\
\text { namic } \\
\text { damping }\end{array}$ \\
\hline Parked & FA & $1.0 \%-3.0 \%$ & $0.2 \%-1.5 \%$ & $0.08 \%-0.24 \%$ & $0.17 \%-1.30 \%$ & $0.11 \%-0.39 \%$ \\
\hline Operational & FA & $5.0 \%-10.0 \%$ & $0.2 \%-1.5 \%$ & $4.0 \%-8.0 \%$ & $0.17 \%-1.3 \%$ & $0.11 \%-0.39 \%$ \\
\hline Parked & SS & $1.0 \%-3.0 \%$ & $0.2 \%-1.5 \%$ & $0.08 \%-0.24 \%$ & $0.17 \%-1.3 \%$ & $0.11 \%-0.39 \%$ \\
\hline Operational & SS & $1.0 \%-4.77 \%$ & $0.2 \%-1.5 \%$ & $0.08 \%-1.43 \%$ & $0.17 \%-1.3 \%$ & $0.11 \%-0.39 \%$ \\
\hline
\end{tabular}

Structural damping for the whole structure could vary from $0.2 \%$ to $1.5 \%$. Published values for hydrodynamic damping range from $0.11 \%$ to $0.39 \%$ which is low both in 
absolute terms and compared to other sources. Soil damping has been reported in the range $0.17 \%$ to $1.3 \%$. Aerodynamic damping values are reported in a fairly narrow range for parked turbines $(0.08 \%-0.24 \%)$, but for operating turbines these values present large variations due to the influence of wind speed, rotor speed and vibration direction (FA and SS). Given the large uncertainties in the available damping values, it is of interest to explore simplified but direct modelling approaches that allow the reported values to be tested.

The values reported in this section warrant a few words of caution. First, in principle, for a linear dynamic system, the damping is a property of the system itself and does not depend on the type of excitation used. This means that the damping values quoted for idle turbines should be the same regardless of the means of obtaining them (e.g. boat impact or rotor stop). However, when aerodynamic damping is present, the applied wind force is coupled with damping mechanism (which makes it nonlinear) so the use of standard linear damping identification techniques is only a convenient expedient. Second, the way the data is processed to identify the damping may affect the results. For instance, if the force applied excites significantly more than one mode, then the identification technique used should involve some spectral analysis (e.g. FFT or wavelet) or the damping estimations may be inaccurate. However, in most of the published papers, the vibration response seems to be clearly dominated by the first bending mode so the values reported above should be considered as first mode damping ratios.

The models proposed in the remainder of the paper also focus on prediction the first bending mode damping ratio.

\section{Numerical modelling approach}

\subsection{Description of the OWT Finite Element model}

In this second part, the behaviour of a monopile-supported OWT was modelled using a bespoke decoupled finite element model. 'Decoupled' here means that the tower FE model will predict the dynamic response of the system but does not include the wind/rotor aerodynamic interaction simultaneously. Decoupled models in this sense have been used to save computational time (e.g. [22][35]). The model is based on the widely used 5MW Reference Offshore Wind Turbine published by NREL [36]. The schematic is shown in Figure 1, and the basic properties of the this turbine are listed in Table 2. A 
lumped mass at the top of the tower represents the Rotor-Nacelle Assembly (RNA). The tower/monopile is modelled with an assembly of 39 2D Euler-Bernoulli elements. The discretisation of the tower followed the segmentation in the data provided for the turbine and a smaller mesh size was used for the monopile. A convergence study (results not shown) confirmed that this element size was adequate. The general expression for the system equation of motion is:

$$
\mathbf{M} \ddot{\mathbf{u}}(t)+\mathbf{C u}(t)+\mathbf{K u}(t)=\mathbf{F}(t),
$$

where $\mathbf{M}, \mathbf{K}$ and $\mathbf{C}$, stand for the mass matrix, stiffness matrix and damping matrix respectively, $\mathbf{u}(t)$ is the displacement vector, and $\mathbf{F}(t)$ is the external force vector. Given the section and material properties of tower over height the mass and stiffness matrices were formed.

The damping calculations from each source will be described in detail in turns in the following sections but there are broadly two approaches. For aerodynamic and hydrodynamic dampings, the dissipation models produced directly a modal damping factor which was calculated with a separate MATLAB program. In this case the FE model was only used to compute the modal properties of the system (see Section 3.2) and a model was fixed at mudline to avoid having to choose a particular soil profile. This is consistent with usual practice in published studies. In the second approach, relevant for soil damping, nonlinear springs and dashpots in parallel were included below the mudline to model the SSI which contributed to the damping and stiffness matrices in Equation (2). The overall modal damping in the system was then extracted through time history analysis. This is described in more detail in Section 3.3.

Structural damping is made of many different mechanisms (material losses, joint friction etc.) which cannot be modelled straightforwardly from first principles. Hence this damping was not considered any further in this paper. However any assumed value could easily be added to a finite element model for instance using a Rayleigh damping matrix in Equation (2). 


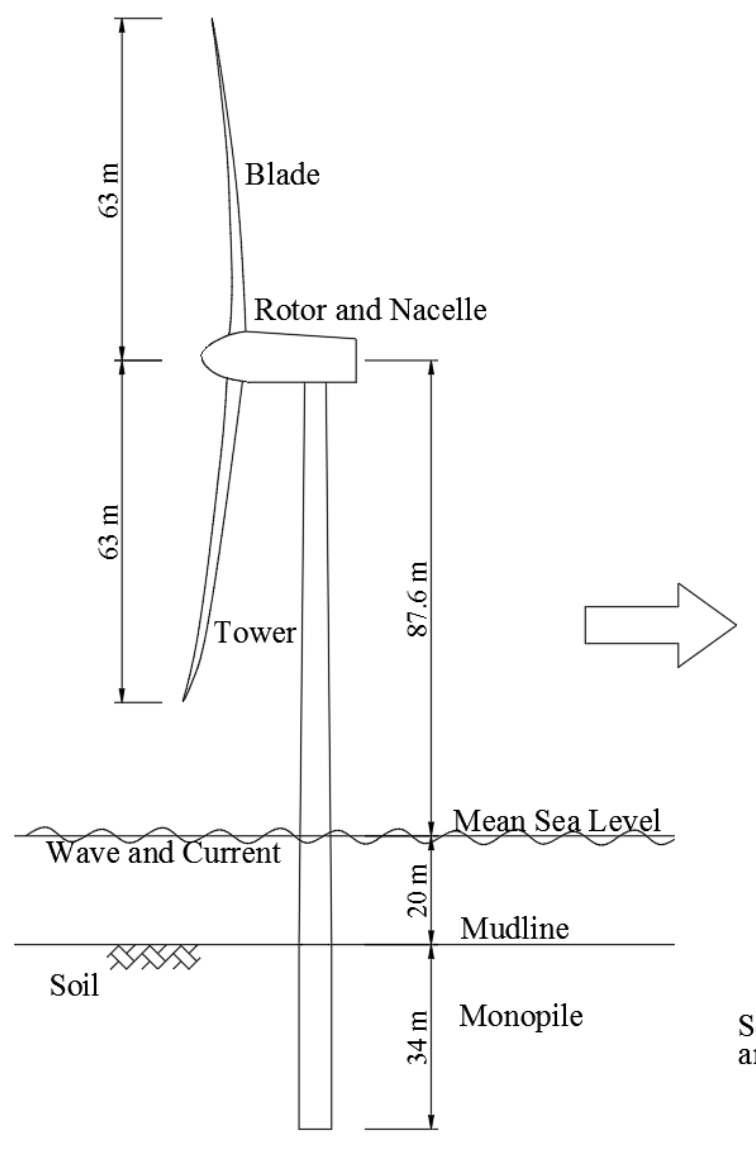

(a) Real 5MW Reference Turbine

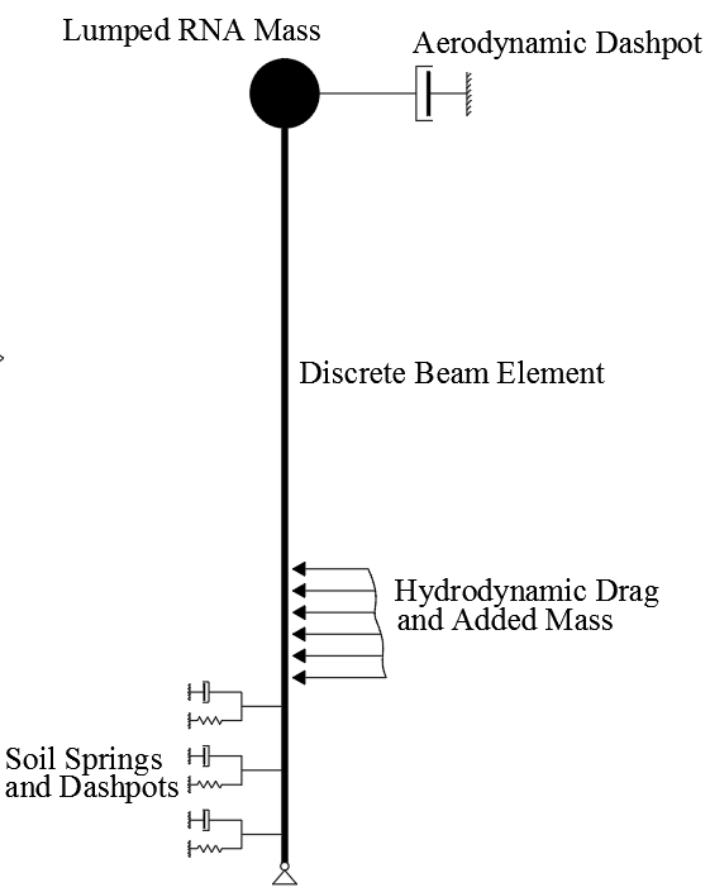

b) Numerical Model

Figure 1 Schematics for (a) 5MW reference turbine and (b) corresponding numerical model

Table 2 Basic Properties of NREL 5MW Reference OWT

\begin{tabular}{|l|c|}
\hline Rotor Diameter, $R$ & $126 \mathrm{~m}$ \\
\hline Hub Height from MSL & $87.6 \mathrm{~m}$ \\
\hline Water Depth, $h$ & $20 \mathrm{~m}$ \\
\hline Tower Diameter, $D$ & $3.87-6.00 \mathrm{~m}$ \\
\hline Tower Thickness, $t$ & $19-60 \mathrm{~mm}$ \\
\hline Monopile Diameter, $D_{\text {Pile }}$ & $6 \mathrm{~m}$ \\
\hline Monopile Thickness, $t_{\text {Pile }}$ & $90 \mathrm{~mm}$ \\
\hline Lumped Mass at Top & $3.5 \times 10^{5} \mathrm{~kg}$ \\
\hline Natural Frequency for Model Fixed at Mudline & $0.27 \mathrm{~Hz}$ \\
\hline Natural Frequency for Model considering SSI & $0.24-0.25 \mathrm{~Hz}$ \\
\hline
\end{tabular}




\subsection{Modal analysis}

Modal damping factors were related to the system properties by writing the equation of motion in modal coordinates. Although standard, this is briefly described here to introduce the notation which is used later. Pre-multiplying Equation (2) with the transpose of the ith mode shape vector $\boldsymbol{\Phi}_{i}^{T}$, the equation of motion becomes:

$$
\bar{m}_{i} \ddot{\alpha}_{i}(t)+\bar{c}_{i} \dot{\alpha}_{i}(t)+\bar{k}_{i} \alpha_{i}(t)=\bar{f}(t),
$$

where $\bar{m}_{i}, \bar{k}_{i}, \bar{c}_{i}$ are respectively the modal mass, stiffness and damping coefficients, and $\alpha_{i}(t)$ is the ith modal coordinate. $\bar{f}_{i}(t)$ is a generalised external force defined as

$$
\bar{f}_{i}(t)=\boldsymbol{\Phi}_{i}^{T} \mathbf{F}(t) .
$$

Rearranging Equation (3) and introducing the modal frequency $\omega_{i}$ and the modal damping ratios $\zeta_{i}$, the equation of motion for the ith mode becomes

$$
\ddot{\alpha}_{i}(t)+2 \zeta_{i} \omega_{i} \dot{\alpha}_{i}(t)+\omega_{i}^{2} \alpha_{i}(t)=\frac{\boldsymbol{\Phi}_{i}^{T} \mathbf{F}(t)}{\bar{m}_{i}} .
$$

In most cases, the contribution from higher modes is negligible so the quantities of interest are the fundamental frequency $\omega_{1}$, the corresponding damping ratio $\zeta_{1}$ and the first modal mass $\bar{m}_{1}$. If a 3D model of the turbine is available then the FA and SS modal properties might be slightly different as the two corresponding rotor moments of inertia associated are quite different. In this case, the relevant values for $\bar{m}_{1}$ and $\omega_{1}$ should be used in the subsequent calculations.

\subsection{Time history analyses and damping identification}

Whenever the damping models did not produce damping ratios directly (i.e. the damping ratios were not calculated from equations based on modal analysis such as Equation (5)), time domain analyses were conducted by implementing the numerical integration scheme HHT- $\alpha$ [37] which is a generalised version of the Newmark- $\beta$ method. The nonlinear stiffness and damping coefficients of the soil springs were directly added to the stiffness and damping matrices at the beginning of each time step. The accuracy of this method was checked by comparing the model with a convergence algorithm (Newton-Raphson method), and good agreement was found.

To extract damping factors from the simulated response time histories, a decaying oscillation was triggered in the system by assigning an initial displacement or acceleration 
to the tower top causing a transient response superimposed on the steady-state behaviour. A sonogram FFT technique was then used on the transient decay so that the damping of the first mode could be isolated [38]. In principle this damping identification technique assumes that the underlying dissipation mechanism is linear. This is not quite the case for the soil model used here however the shape of the decays obtained were close enough to those of a linear system to justify the use of the method.

\section{Aerodynamic damping}

\subsection{Theoretical derivation of the aerodynamic force}

Aerodynamic damping is caused by the drag forces experienced by the oscillating rotor in the surrounding air flow. In our model, the rotor is represented by a lumped mass at the top of the beam representing the tower. The motion of the entire rotor is assumed to be consistent with that of the beam tip in terms of translation but not rotation. In BEM theory, the resultant aerodynamic force applied to the hub can be obtained by introducing a relative wind speed experienced by each blade element and summing up the element thrust component. These resultant forces and relative speeds are different in the fore-aft and side-side direction so these two cases must be dealt with separately.

\section{Fore-aft aerodynamic damping}

It is assumed that the rotor is rotating in its own plane with the angular speed of $\omega$ and exposed to a uniform steady incoming wind field of average speed $V_{W x}$, as shown in Figure 2. When the rotor does not oscillate, the three blade elements at radius $r$ from the hub, with thickness $d r$ experience an elemental thrust $\left.d T\right|_{V_{x}}$ :

$$
\left.d T\right|_{V_{x}}=\frac{1}{2} \rho_{a} N_{b} \frac{V_{x}^{2}}{\sin ^{2} \phi} c C_{n} d r
$$

where $V_{x}$ is the axial component of the relative wind speed felt by the blade element; $N_{b}$ is the number of blades, $\rho_{a}$ is the air density, $c$ is the chord length, $C_{n}=$ $C_{l} \cos \phi+C_{d} \sin \phi$, with $C_{l / d}$ the lift/drag coefficients and $\phi$ is the sum of the attack, pitch and twist angles. If the tower top moves in the fore-aft direction with a velocity $\dot{x}$, the element now feels a relative velocity $V_{x R e l}$ :

$$
V_{x R e l}=V_{x}-\dot{x} .
$$



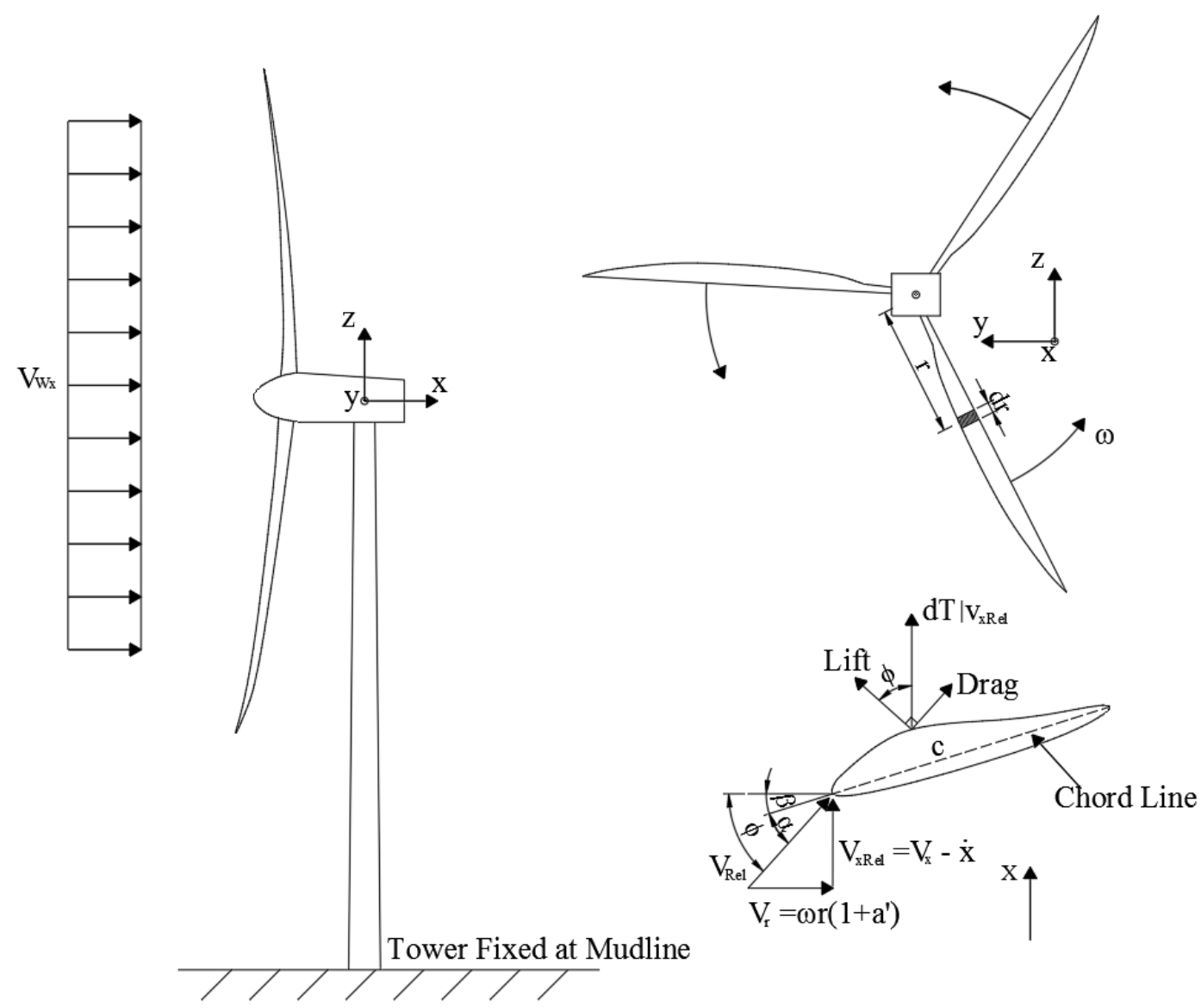

Figure 2 Blade element forces for fore-aft vibration.

Assuming that $\dot{x}$ is a small compared to $V_{x}$ and that it is the same throughout the rotor (the rotor remains parallel to itself), $\left.d T\right|_{V_{x R e l}}$, the thrust felt by the three blade elements under a relative speed of $V_{x R e l}$, can be obtained from $\left.d T\right|_{V_{x}}$ through a first-order Taylor expansion:

$$
\begin{gathered}
\left.d T\right|_{V_{x R e l}}=\left.d T\right|_{V_{x}}+\left.d T^{\prime}\right|_{V_{x}} \cdot\left(V_{x R e l}-V_{x}\right) \\
=\left.d T\right|_{V_{x}}-\frac{d(d T)}{d V_{x}} \dot{x} .
\end{gathered}
$$

From this derivation, it is clear that the aerodynamic damping will come into the equation of motion of the tower through the viscous term $\frac{d(d T)}{d V_{x}} \dot{x}$. The objective is to calculate this term and obtain the resultant thrust.

Assuming that $d T$ is a function of $V_{x}$ and $\phi$, and that $\phi$ is a function of $V_{x}, \frac{d(d T)}{d V_{x}}$ can be calculated as

$$
\frac{d(d T)}{d V_{x}}=\frac{\partial(d T)}{\partial V_{x}}+\frac{\partial(d T)}{\partial \phi} \frac{d \phi}{d V_{x}}
$$


In Eq. 9, $\frac{\partial(d T)}{\partial V_{x}}$ and $\frac{\partial(d T)}{\partial \phi}$ can be obtained by differentiating Equation (6):

$$
\begin{gathered}
\frac{\partial(d T)}{\partial V_{x}}=\rho_{a} N_{a} \frac{V_{x}}{\sin ^{2} \phi} c C_{n} d r, \\
\frac{\partial(d T)}{\partial \phi}=\frac{1}{2} \rho_{a} N_{a} V_{x}^{2} \frac{\frac{d C_{n}}{d \phi} \sin ^{2} \phi-2 \sin \phi \cos \phi C_{n}}{\sin ^{4} \phi} c d r
\end{gathered}
$$

where

$$
\begin{aligned}
& \frac{d C_{n}}{d \phi}=\frac{\partial C_{l}}{\partial \phi} \cos \phi+\frac{\partial C_{d}}{\partial \phi} \sin \phi+C_{d} \cos \phi-C_{l} \sin \phi \\
& \frac{d C_{t}}{d \phi}=\frac{\partial C_{l}}{\partial \phi} \sin \phi-\frac{\partial C_{d}}{\partial \phi} \cos \phi+C_{l} \cos \phi+C_{d} \sin \phi
\end{aligned}
$$

To obtain an expression for $\frac{d \phi}{d V_{x}}$, the following two equations can be used:

$$
\begin{aligned}
& \tan \phi=\frac{V_{x}}{\omega r\left(1+a^{\prime}\right)}, \\
& a^{\prime}=\frac{1}{\frac{4 \sin \phi \cos \phi}{\sigma C_{t}}-1} .
\end{aligned}
$$

Differentiating Equation (14) with respect to $V_{x}$ and Equation (15) with respect to $\phi$ gives:

$$
\frac{d \phi}{d V_{x}}=\frac{1}{\omega r\left(\frac{1+a^{\prime}}{\cos ^{2} \phi}+\frac{d a^{\prime}}{d \phi} \tan \phi\right)}
$$

and

$$
\frac{d a^{\prime}}{d \phi}=\frac{-4\left(\cos 2 \phi C_{t}-\frac{d C_{t}}{d \phi} \sin \phi \cos \phi\right)}{\sigma C_{t}^{2}\left(\frac{4 \sin \phi \cos \phi}{\sigma C_{t}}-1\right)^{2}}
$$

Equations (10), (11) and (16) can be substituted into Equation (9), and the resulting expression for $\frac{d(d T)}{d V_{x}}$ can be used to obtain $\left.d T\right|_{V_{x R e l}}$ in Equation (8) which is the aerodynamic force subjected by the three blade elements. For simplicity, denote

$$
\begin{gathered}
A \cdot d r=\frac{\partial(d T)}{\partial V_{x}}, \\
B \cdot d r=\frac{\partial(d T)}{\partial \phi} \frac{d \phi}{d V_{x}} .
\end{gathered}
$$

So, the thrust felt by the three blade elements is:

$$
\left.d T\right|_{V_{x R e l}}=\left.d T\right|_{V_{x}}-(A+B) d r \cdot \dot{x} .
$$

The total thrust felt by the rotor is: 


$$
F_{x}=\left.\int_{0}^{R} d T\right|_{V_{x R e l}}=\left.\int_{0}^{R} d T\right|_{V_{x}}-\int_{0}^{R}(A+B) d r \cdot \dot{x},
$$

where $R$ is the radius of the rotor. Equation (21) shows that the total thrust can be divided in two parts: the first part $\left.\int_{0}^{R} d T\right|_{V_{x}}$ represents the static force caused by the steady wind on a rigid structure; the second part $-\int_{0}^{R}(A+B) d r \cdot \dot{x}$ is the aerodynamic damping force proportional to the oscillating velocity of the entire rotor. Applying the total thrust on the top of the tower, the turbine model is able to account for the aerodynamic damping at a given rotor speed, wind speed and pitch angles without coupling the tower and the rotor models. The damping part $\int_{0}^{R}(A+B) d r$ can be used to calculate the damping ratio with the following equation:

$$
\zeta_{\text {Aero_ } F A}=\frac{\int_{0}^{R}(A+B) d r}{2 \bar{m}_{1} \omega_{1}} .
$$

\section{Side-side aerodynamic damping}

Following a similar procedure, the total side-side aerodynamic force felt by the rotor can be expressed as:

$$
F_{y}=\frac{N_{b}}{2} \int_{0}^{R}\left(A^{\prime}+B^{\prime}\right) d r \cdot \dot{y}
$$

where $A^{\prime}$ and $B^{\prime}$ are derived in Appendix $\mathrm{A}$ and $\dot{y}$ is the side-side velocity of the tower top. Using modal analysis, the side-side damping ratio can be expressed as:

$$
\zeta_{\text {Aero_SS }}=\frac{-\frac{N_{b}}{2} \int_{0}^{R}\left(A^{\prime}+B^{\prime}\right) d r}{2 \bar{m}_{1} \omega_{1}} .
$$

\subsection{Aerodynamic damping comparison}

Equations (22) and (24) were implemented in MATLAB and coupled with the modal properties obtained from the FE model. These results are here compared to FAST simulations obtained with identical settings for validation purposes. For a range of steady wind speeds, FAST simulations were run, leaving the control module in FAST to alter the pitch angles and the rotating speed of rotor to achieve the desired power output. Figure 3 shows the relationship obtained this way between rotor speed, pitch and wind speed for the same NREL 5MW turbine. 


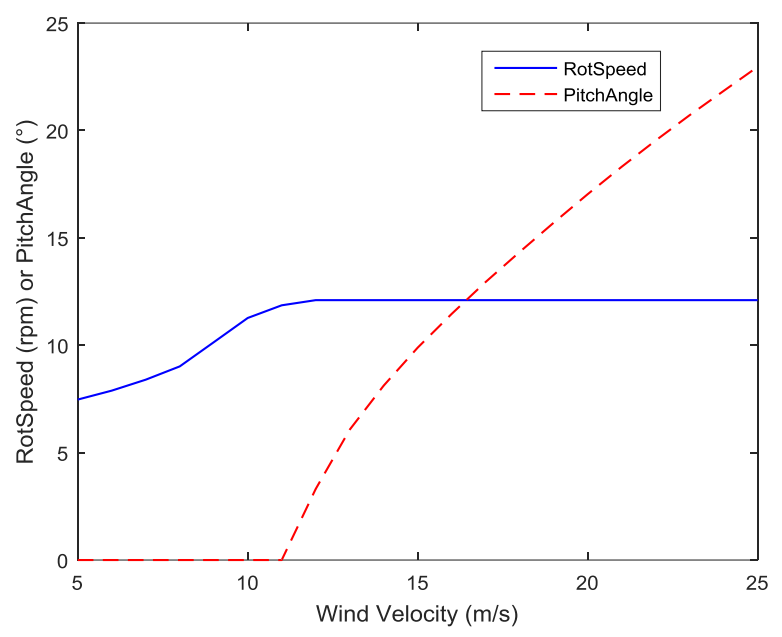

Figure 3 Steady-state pitch angles and rotor speeds

for different wind speed in the FA direction

For each average wind speed, an initial displacement of 1 metre was given to the tower top and FAST was used to calculate time series of the tower top response. From these time series damping ratios were estimated as described in Section 3.3.

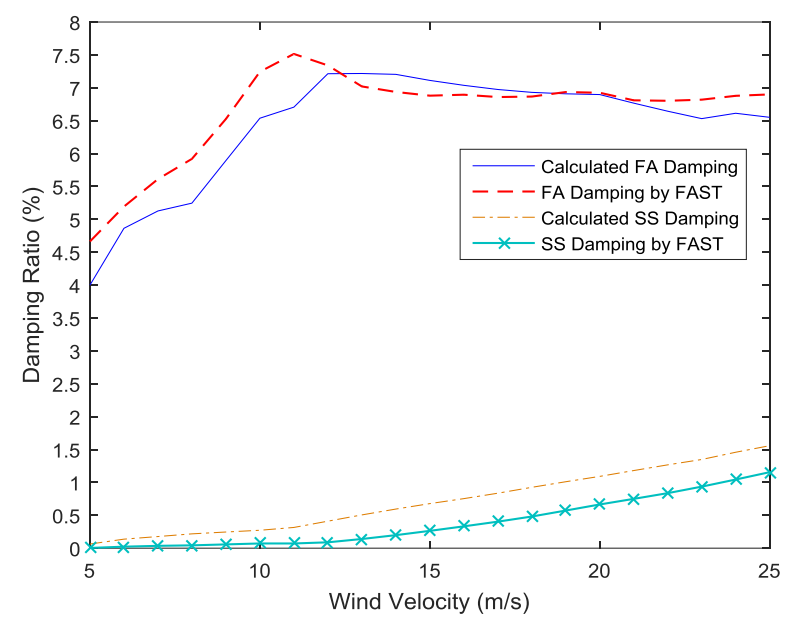

Figure 4 Comparison of FA and SS damping ratios for varying wind speeds obtained from BEM method and from FAST.

Figure 4 show the comparison of damping ratios in the fore-aft and side-side directions (respectively) using FAST and the proposed modelling approach for varying wind speeds. The FA and SS degrees of freedom were decoupled in the FAST simulations to mirror the BEM modelling assumptions. For example, when looking at the FA damping, the fore-aft degree of freedom (DOF) was on but the side-side DOF was off and vice-versa. In the FA direction, good agreement is found between FAST and BEM results. Figure 4(a) confirms that for the turbine modelled here, FA aerodynamic damping 
increases from $5 \%$ at low wind speed to $7 \%$ at the rated speed after which it remains broadly constant. This range is in full agreement with the results reported in the literature. In the SS direction, the damping ratios obtained from BEM and FAST follow similar trends and the damping ratios are much lower than in the FA direction and they increase steadily from $0.2 \%$ to $1.2-1.6 \%$ at the top wind speed in the operating range. BEM results appear systematically higher than those from FAST by a fraction of a percent. Further research currently under way suggests that this difference is associated with the rotation of the top which is ignored here (but not in FAST). In fact, the tilting and sideway motions of the tower mean that the induced velocity seen by blade elements differs from the one we calculate and the wake flow angle is less than $90^{\circ}$ to the rotor plane [39]. This phenomenon generates unbalanced resultant forces in the SS direction at the rotational frequency of the rotor which cause the tower to oscillate. In turn, the SS motion causes a harmonic excitation on the FA motion. FAST simulations not shown here suggest that this coupling does not change much the overall range of damping values obtained but it can affect the dependence on the wind speed. In summary, the proposed aerodynamic model based on BEM theory is adequate to model the aerodynamic damping in the FA direction and gives a reasonable estimate in the SS direction. However, it cannot capture more detailed effects related to coupling which appear to affect the dependency of the damping ratio on the wind speed above the rated speed of the turbine.

\section{Hydrodynamic damping}

In this section hydrodynamic damping is quantified directly on the basis of classical theories and numerical simulations. As a simple but representative model, a flexible cantilevered cylinder immersed in water up to MSL is considered and subjected to wave excitation as shown in Figure 5. There are mainly two approaches to calculate the hydrodynamic forces resulting from the interaction between the cylinder and the seawater: (1) through Morison's equation or (2) potential flow theory. Morison's equation deals with the viscous effects when flow separation is significant, while potential flow theory accounts for the diffraction and radiation phenomena.

The physics of the water-cylinder interaction can be very different depending on the value of key non-dimensional parameters. The most relevant ones here are the Keule- 
gan-Carpenter number $K C$, the Stokes parameter $\beta_{\text {Stokes }}$ and the diffraction factor $D / \lambda$ where $\lambda$ is the wavelength and $D$ the diameter of the pile. By definition, $K C=2 \pi \hat{x} / D$ where $\hat{x}$ is the amplitude of the sideway motion, and $\beta_{\text {Stokes }}=$ $D^{2} / v_{w} T$ where $v_{w}$ is the kinematic viscosity of water and $T$ is the oscillation period. In the case of a large monopile submerged in water, the $K C$ number is in the range of low values, around $10^{-2}$, while the Stokes parameter and the diffraction factor are relatively large. These parameter values indicate that the flow separation tends to be small but not insignificant while diffraction and radiation are the dominant phenomena [40]. This is supported by Johanning et al. [41], who found that the damping of an oscillating cylinder can be influenced by both the viscous damping and radiation damping caused by hydrodynamic forces. In this paper, hydrodynamic damping is estimated by modelling the viscous (Morison's equation) and wave (potential theory) contributions separately.

In addition to the two dissipative effects mentioned above, the added mass coming from the volume of water moving together with the cylinder can influence the mass distribution of the monopile under mean sea level (MSL). The added mass tends to reduce the damping ratio, but this effect has been found to be negligible [42]. The low relative acceleration in the submerged tower (since this part is near to the clamped end) results in a very small effect on the frequency and dynamic response of the whole system. Therefore, in the subsequent calculations, the modal mass does not account for the hydrodynamic added mass.

Both for viscous and radiation damping, the wave height and wave period were set to $6 \mathrm{~m}$ and 10s for all calculations and the wave velocities were calculated using linear wave theory [43]. This single combination of wave period and wave height was selected for simplicity, but is representative of the most common sea states [44].

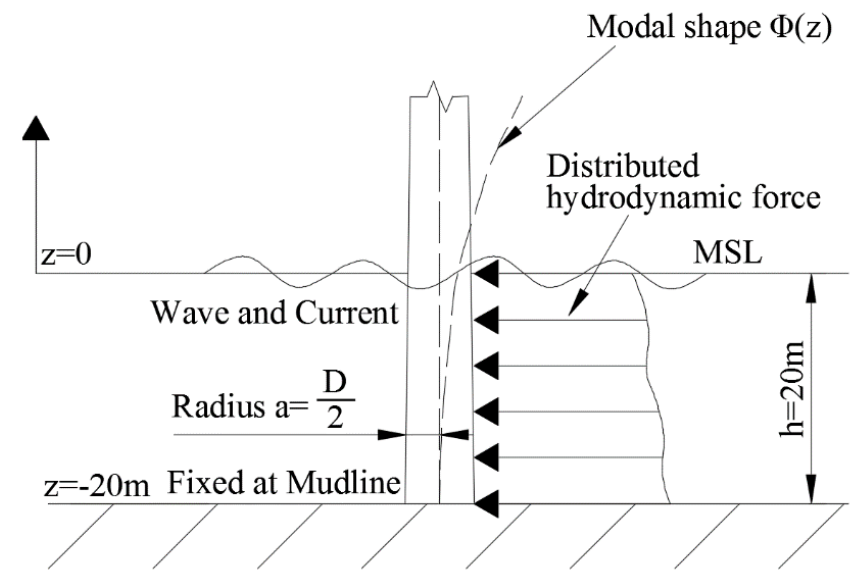


Figure 5 Schematic of hydrodynamic forces on OWT substructure

\subsection{Viscous damping}

For an oscillating cylinder excited by waves, Morison's equation gives the forces on a strip of cylinder at depth $z$ :

$$
\begin{gathered}
F_{\text {Morison }}=\frac{1}{2} \rho_{w} D C_{d}\left|\dot{u}_{w}-\dot{v}_{\text {tower }}\right|\left(\dot{u}_{w}-\dot{v}_{\text {tower }}\right) \\
+\frac{\pi}{4} \rho_{w} D^{2} C_{a}\left(\ddot{u}_{w}-\ddot{v}_{\text {tower }}\right)+\frac{\pi}{4} \rho_{w} D^{2} \ddot{u}_{w}
\end{gathered}
$$

where $u_{w}$ is the velocity of water particles, $v_{\text {tower }}$ is the velocity of the vibrating tower under MSL, $C_{d}$ is the drag coefficient, $C_{a}$ is the added mass coefficient and $\rho_{w}$ is the density of water. The damping term is quadratic in the relative velocity and influenced by the drag coefficient $C_{d}$. The value of $C_{d}$ is mainly dependent on the $K C$ number and the Stokes parameter [45][46]. According to Bearman and Russell, for low values of $K C$ number (up to 5) and high values of Stokes number (in the order of $\left.10^{5}\right), C_{d}$ can be obtained by the following equation:

$$
C_{d}=\frac{2 \times 26.24}{K C \sqrt{\beta_{\text {Stokes }}}}+0.08 \mathrm{KC},
$$

where the second term in this equation can be neglected for even smaller $K C$ numbers. As KC depends on the vibration amplitude, $C_{d}$ could be made to vary along the depth of the cylinder according to Equation (26). This was tested and the damping did not show much sensitivity to $C_{d}$ so $C_{d}=1$ was used throughout, based on the velocity amplitude of the pile at MSL obtained from simulation of the 5MW reference turbine and recommendations from past study (e.g. [11]).

The viscous drag term in Morison's equation varies along the immersed cylinder as a result of the non-uniform distribution of vibration accelerations and velocities. Following [47] and after rearranging, the damping ratio for a single pile subjected to viscous drag can be written as:

$$
\zeta_{\text {Hydro,Vis }}=\frac{\int_{-h}^{0} \frac{1}{2} \rho_{w} D C_{d} \sqrt{\frac{8}{\pi}} \sigma_{r}(z) \Phi^{2}(z) d z}{2 \bar{m} \omega_{n}},
$$

where $\sigma_{r}(z)$ is the root mean square of the relative speed between the water wave particle and the pile (and other notation was defined in 3.2). For large OWTs, the velocity of the pile can be estimated by $\frac{2 \pi \hat{x}}{T} \sim 0.1 \mathrm{~m} / \mathrm{s}$. This is much smaller than the wave particle velocity (usually above $10 \mathrm{~m} / \mathrm{s}$ ), so $\sigma_{r}(z)$ can be replaced by the root mean 
square of the particle velocity. Usually, the wave profile can be generated using specific spectra, so $\sigma_{r}(z)$ could be calculated from these spectra.

\subsection{Radiation damping}

According to linear potential flow theory, when only wave radiation is considered (i.e. ignoring the fluid viscosity), wave-structure interaction produces two kinds of forces: those produced by the incoming wave field on a rigid cylinder and those due to the motion of the structure in a disturbance-free fluid. Only the latter gives rise to dissipation and can be expressed in Equation (5) by

$$
\bar{f}(t)=-\int_{-h}^{0} C_{R a d} d z \cdot \dot{\alpha}(t),
$$

where $C_{R a d}$ is the radiation factor, the derivation of which is shown in Appendix B based on the study by [48]. Substituting Equation (28) into Equation (5) and rearranging, gives a radiation damping ratio:

$$
\zeta_{H y d r o, R a d}=\frac{\int_{-h}^{0} C_{R a d} d z}{2 \omega_{1} \bar{m}} .
$$

\subsection{Hydrodynamic damping comparison}

For the model of the 5MW reference OWT, the viscous damping ratio calculated by Equation (27) is $0.0006 \%$, while the radiation damping ratio according to Equation (29) is $0.007 \%$, giving a total hydrodynamic damping ratio of $0.0076 \%$. This result is much smaller than what has been reported in past research papers such as [11] which suggests the viscous damping ratio is around $0.004 \%$ and radiation damping ratio is $0.12 \%$. This difference is explained by the size of the structure which is much larger in our case than those considered in the literature. In [48] from which the $0.12 \%$ value for radiation damping value seems to originate, the diameter of the pile considered was $1.2 \mathrm{~m}(4 \mathrm{ft})$ and the structure was much shorter above MSL (so the dynamics of the structures were very different). When their dimensions are used in the equations presented in this section, $0.11 \%$ is obtained confirming the soundness of the calculation and the effect of the size. To explore this further, plausible dimensions for tower height, water depth and pile thickness (based on the turbine scaling equations proposed in [49]) were estimated so that damping values could be calculated for various turbine sizes. The results are 
shown in Figure 6, confirming that both viscous and radiation damping are always very small for large diameter rotors.

The conclusion from these models is that hydrodynamic damping in OWTs is much smaller than usually assumed and for all practical purposes could be safely ignored.

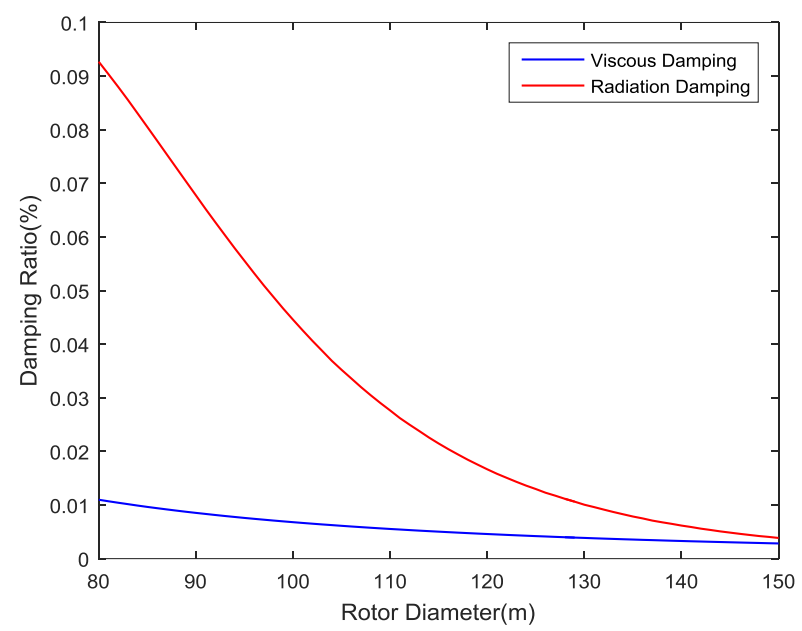

Figure 6 Variation of viscous and radiation damping ratios for different turbine sizes

\section{Soil damping}

The soil surrounding the monopile foundation can dissipate vibrational energy in two ways: (1) the motion of the pile can cause waves to radiate away from the pile into the ground (radiation damping), and (2) hysteretic material damping can occur as the soil is being cyclically stressed [26][50]. Radiation damping depends on the frequency $\omega$ of the external excitation. The non-dimensional parameter $a_{0}=\omega D / V_{s}$ can be used to assess the significance of the wave phenomenon. It compares the order of magnitude of the pile lateral velocity and the shear wave velocity of the soil stratum $V_{s}$. The shear wave velocity ranges from $150 \mathrm{~m} / \mathrm{s}$ to $300 \mathrm{~m} / \mathrm{s}$ depending on the soil type (see Table 4 below). For a monopile-supported OWT, the forces experienced by the soil will usually have a low frequency around the first natural frequency (less than $1 \mathrm{~Hz}$ ) as the response of the system is dominated by the first vibration mode. In these conditions, $a_{0}$ can be expected to be of the order of $10^{-2}$, indicating that the behaviour of the soil is largely quasi-static so that radiation damping for a monopile-supported OWT can be ignored [28].

\subsection{Soil model}




\section{Stiffness and damping coefficients for soil springs and dashpots}

Soil-pile interaction can be modelled using different approaches such as linear or nonlinear Winkler's springs or finite element solid modelling. For design purposes, p-y curves which capture the nonlinear displacement-force relationship in the soil, are frequently used. In the present study, a combination of classic p-y curves and the study by Gazetas and Dobry [28] is adopted. The soil is represented by a series of horizontal springs and dashpots in parallel from the mudline to bottom of the pile. The stiffness of the springs was obtained from $\mathrm{p}-\mathrm{y}$ curves while the dashpot coefficients representing the soil material damping were calculated following Gazetas and Dobry [28]:

$$
c_{m}=2 k \frac{\beta_{m}}{\omega},
$$

where $\beta_{m}$ represents the material damping ratio, $k$ is the secant modulus defined as the ratio between the static local soil reaction for a unit length of pile and the corresponding local pile deflection, $\omega$ is the circular frequency of the excitation at the pile head. The magnitude of material damping ratio for a soil stratum is related to the shear strain in the soil, and can be influenced by the properties of soil such as plastic index and mean effective confining stress. Darendeli [31] proposed an analytical model to generate material damping curves for various values of the over consolidation ratio (OCR) and plasticity index (PI). This study adopts this method and uses the coefficients for sand and clay from Southern California provided by Darendeli as the parameters he uses are somewhat idiosyncratic and not available from standard data tables. However Darendeli's data as well as other experimental studies in soil damping [30] show that the curves produced using his formulae are representative of many soil types.

Figure 7 shows the relationship between the material damping ratio and soil shear strain for clay in which the damping depends on the Plasticity Index to some extent so various curves were plotted for different PI values. The PI values used for the clay soils is shown in Table 3. Sandy soils (not shown) have a very similar relationship between material damping ratio and shear strain but their PI should be 0 . Soil material damping also depends slightly on the OCR but in the context of offshore wind turbines, OCR should be 1 and this value was used for all soil types considered in this study. 


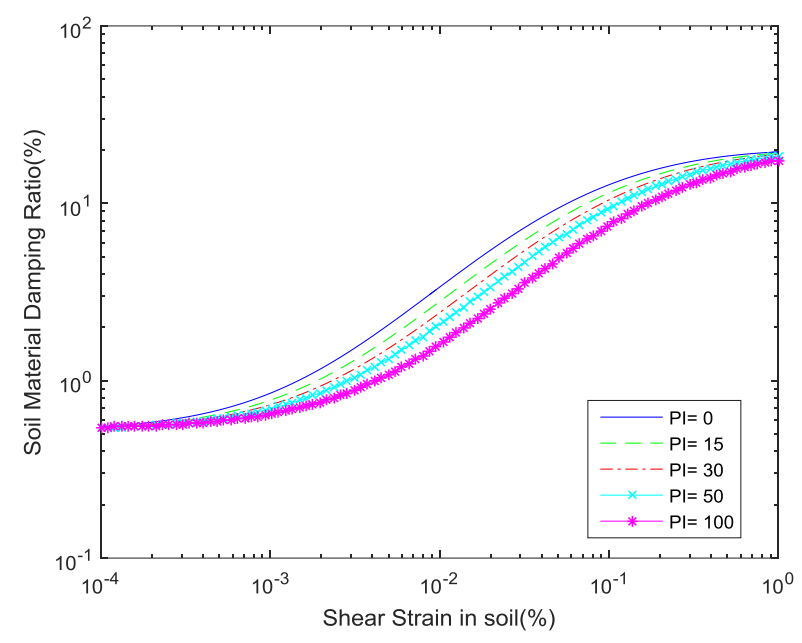

Figure 7 Relationship between material damping ratio and shear strain in clay for different PI values.

Following [28], the shear strain $\gamma_{e}$ can be determined by

$$
\gamma_{e}=\frac{1+v}{2.5 D} y_{A m p}(z, t)
$$

where $y_{A m p}(z)$ is the oscillating amplitude of the pile in the horizontal direction, $v$ is Poisson's ratio for the soil stratum, and $D$ is the diameter of the pile. $k$ is determined from the non-linear $\mathrm{p}-\mathrm{y}$ curves:

$$
k=\frac{p(z, t)}{y(z, t)} .
$$

The soil around a wind turbine experiences some cyclic strain on top of a constant strain caused by the mean wind thrust. Whether the total amplitude or simply the dynamic component should be used for $y_{A m p}(z)$ is not obvious. In this study, the amplitude $y_{A m p}$ used to calculate the strain (and subsequently the damping ratio) was half the range of oscillation. An alternative method commonly used in earthquake engineering [30] is to evaluate the damping constant using $65 \%$ of the maximum strain reached in the soil strata during a response time history. Earthquake ground motion does not usually have a static component so it is still not clear how to implement this rule here but this was also tested and found to give very similar results to those obtained using Equation (31).

\section{Soil properties and p-y curves}


Here six soil profiles are selected to investigate the soil damping contribution. Profiles 1-4 are single material soils made of medium clay, stiff clay, medium sand and dense sand respectively. The low stiffness of soft clay and loose sand would significantly reduce the stiffness of the entire system and lead to unrealistic designs, so they were excluded as monolayered soils. Profiles 5 and 6 are layered soil profiles combining a mix of clay and sandy soils respectively. The layered clay profile is a combination of stiff clay, medium clay and soft clay from bottom to pile head with the height of $14 \mathrm{~m}$, $14 \mathrm{~m}$ and $6 \mathrm{~m}$ respectively. The layered sandy profile is a combination of loose sand, medium sand and dense sand from bottom to pile head with the height of $14 \mathrm{~m}, 14 \mathrm{~m}$ and $6 \mathrm{~m}$ respectively which is a typical layered profile based on the data provided in Appendix B in [51]. The different soil profiles used are summarised in Table 3 while the properties of the different soil types are listed in Table 4.

Table 3 Soil profiles definition

\begin{tabular}{|c|c|c|c|c|c|}
\hline Profile 1 & Profile 2 & Profile 3 & Profile 4 & Profile 5 & Profile 6 \\
\hline $\begin{array}{c}\text { medium } \\
\text { clay }\end{array}$ & stiff clay & $\begin{array}{c}\text { medium } \\
\text { sand }\end{array}$ & $\begin{array}{l}\text { dense } \\
\text { sand }\end{array}$ & $\begin{array}{ll}28 m-34 m & \text { Soft clay } \\
14 m-28 m & \text { Medium clay } \\
0 m-14 m & \text { Stiff clay }\end{array}$ & \begin{tabular}{|ll}
$28 m-34 m$ & Loose sand \\
$14 m-28 m$ & Medium sand \\
$0 m-14 m$ & Dense sand
\end{tabular} \\
\hline $\mathrm{PI}=20$ & $\mathrm{PI}=20$ & $\mathrm{PI}=0$ & $\mathrm{PI}=0$ & $\mathrm{PI}=20$ & $\mathrm{PI}=0$ \\
\hline $\mathrm{OCR}=1$ & $\mathrm{OCR}=1$ & $\mathrm{OCR}=1$ & $\mathrm{OCR}=1$ & $\mathrm{OCR}=1$ & $\mathrm{OCR}=1$ \\
\hline
\end{tabular}

Table 4 Mechanical properties of the soil profiles used in the model

\begin{tabular}{|c|c|c|c|c|c|c|c|c|}
\hline & $\begin{array}{l}\text { Soil } \\
\text { type }\end{array}$ & $\begin{array}{c}\text { Density } \\
\gamma \\
\left(1 \times 10^{3}\right. \\
\left.\mathrm{kg} / \mathrm{m}^{3}\right)\end{array}$ & $\begin{array}{l}\text { Undrained } \\
\text { shear } \\
\text { strength } \\
\qquad c_{u} \\
\quad(\mathrm{kPa})\end{array}$ & $\begin{array}{l}\text { Poisson's } \\
\text { ratio } \\
\quad v\end{array}$ & $\begin{array}{c}\text { Friction } \\
\text { angle } \\
\qquad\end{array}$ & $\begin{array}{c}\text { Shear } \\
\text { modulus } \\
G_{S} \\
(\mathrm{MPa})\end{array}$ & $\begin{array}{c}\text { Shear } \\
\text { wave } \\
\text { velocity } \\
V_{S} \\
(\mathrm{~m} / \mathrm{s})\end{array}$ & $\begin{array}{c}\text { Elastic } \\
\text { Moddulus } \\
E_{S} \\
(\mathrm{MPa})\end{array}$ \\
\hline \multirow[t]{3}{*}{ Clay } & $\begin{array}{l}\text { Soft } \\
\text { Clay }\end{array}$ & 1.75 & 30 & 0.45 & - & 43 & 158 & 15 \\
\hline & $\begin{array}{l}\text { Medium } \\
\text { Clay }\end{array}$ & 1.90 & 80 & 0.45 & - & 87 & 214 & 30 \\
\hline & $\begin{array}{l}\text { Stiff } \\
\text { Clay }\end{array}$ & 2.00 & 125 & 0.45 & - & 145 & 269 & 50 \\
\hline Sand & $\begin{array}{l}\text { Loose } \\
\text { Sand }\end{array}$ & 1.75 & - & 0.30 & $33^{\circ}$ & 47 & 164 & 18 \\
\hline
\end{tabular}




\begin{tabular}{|l|l|c|c|c|c|c|c|c|}
\hline $\begin{array}{l}\text { Medium } \\
\text { Sand }\end{array}$ & 1.90 & - & 0.30 & $36^{\circ}$ & 109 & 240 & 42 \\
\cline { 2 - 8 } & $\begin{array}{l}\text { Dense } \\
\text { Sand }\end{array}$ & 2.07 & - & 0.30 & $38^{\circ}$ & 182 & 297 & 70 \\
\hline
\end{tabular}

The SSI effect was modelled using static or cyclic p-y curves recommended in DNV 2014 [52] and API standards [53], and the steps to establish the p-y curves are detailed in Reese and Van Impe [54]. Although it would seem more sensible to use the cyclic curves to represent the SSI of an OWT under long-term excitations, both static and cyclic p-y curves were used to allow comparison with published results. Figure 8 shows the static and cyclic $\mathrm{p}$-y curves for each soil types at the depth $\mathrm{z}=-8.5 \mathrm{~m}$ from mudline. These figures illustrate that, whether static or cyclic p-y curves are used, the forces from the soil plateau vary quickly in sandy soils as the pile moves sideway. By contrast, the behaviour of clay soils is more complex and variable as the lateral displacement of the pile increases. The cyclic p-y curves are recognisably similar to the static ones except that much larger forces can be reached in the cyclic case.

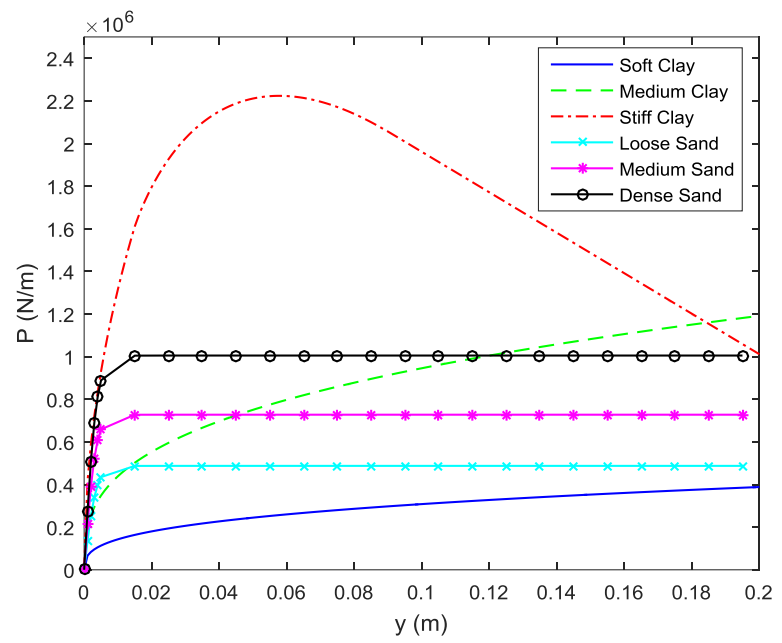

(a)

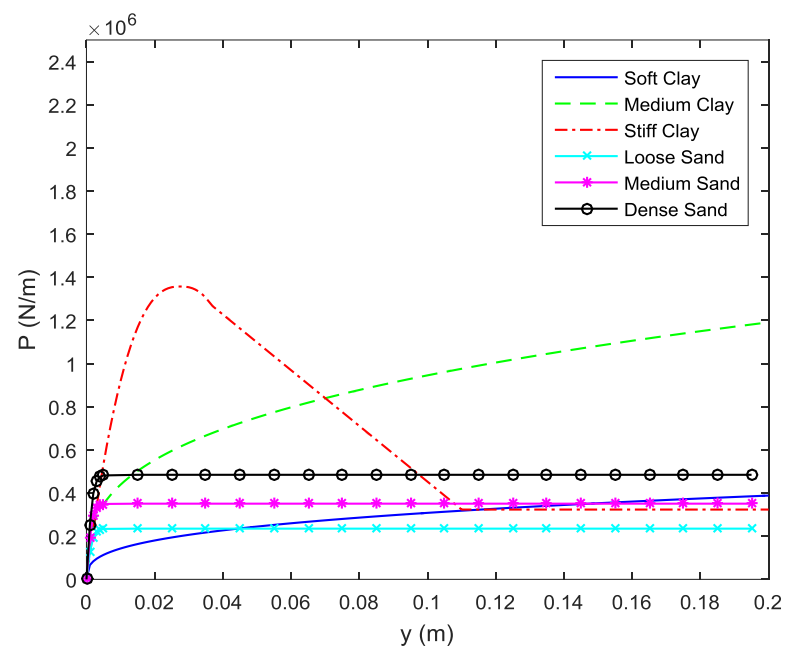

(b)

Figure 8 Static (a) and cyclic (b) p-y curves for different soil types

In this section, the finite element model of the OWT included soil springs and dashpots whose properties were obtained using Equations (30) and (32) respectively. As the response of the soil is strongly strain-dependent, the model was subjected to forces of increasing intensity. This was implemented using thrust time series obtained from FAST with mean speed increasing from $5 \mathrm{~m} / \mathrm{s}$ to $25 \mathrm{~m} / \mathrm{s}$ by $1 \mathrm{~m} / \mathrm{s}$ increments. To avoid the stochastic component of the wind adding noise to the response of the system and 
interfering with the damping calculation, these time series were averaged and the average was used as a static force applied at the tower top in the finite element model. Then the soil damping was calculated from the vibration decay ensuing an applied initial condition of a magnitude consistent with the response of the system to the give force. For these simulations, the control system was kept on in FAST. This enables blade pitching and causes a decrease in aerodynamic force in the FA direction for wind speeds above the rated speed $(12 \mathrm{~m} / \mathrm{s}$ in this case).

\subsection{Soil damping results and discussion}

Figure 9 shows the variation of the damping ratios in the various soils for mean wind speeds ranging from $5 \mathrm{~m} / \mathrm{s}$ to $25 \mathrm{~m} / \mathrm{s}$.

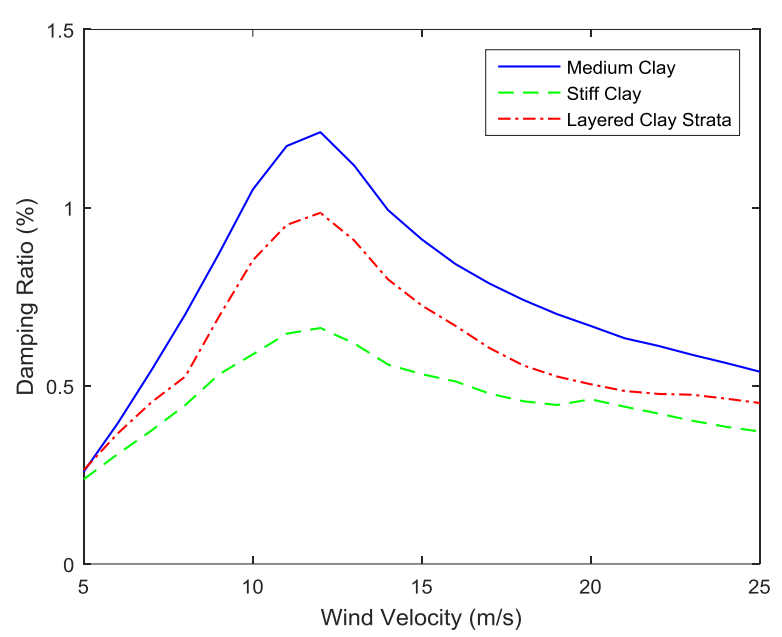

(a)

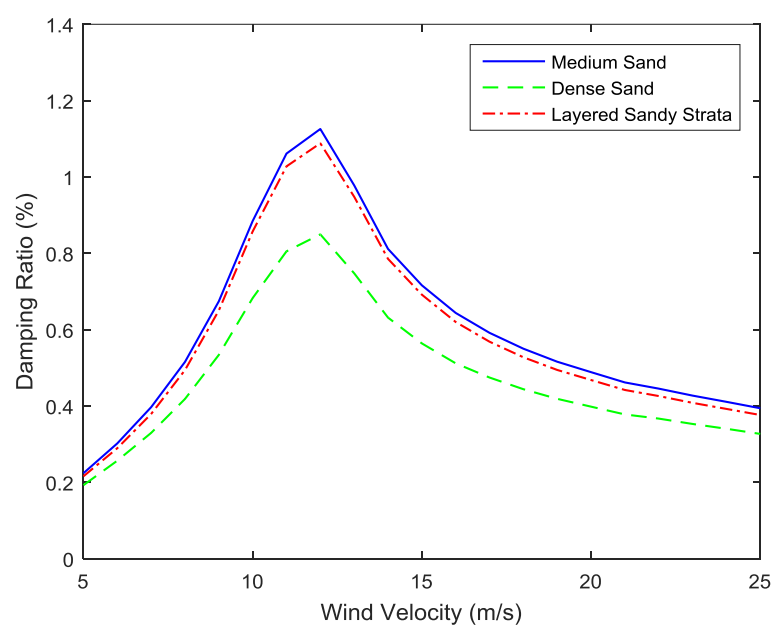

(c)

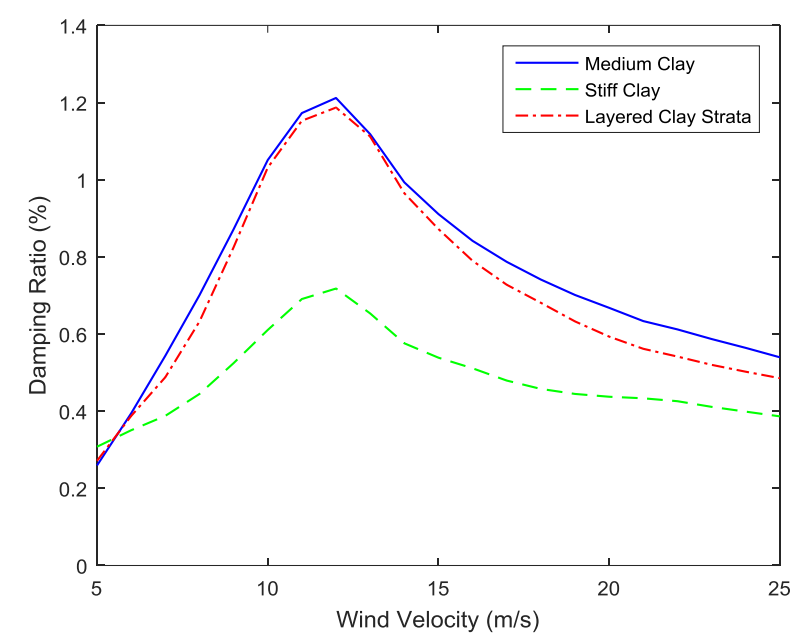

(b)

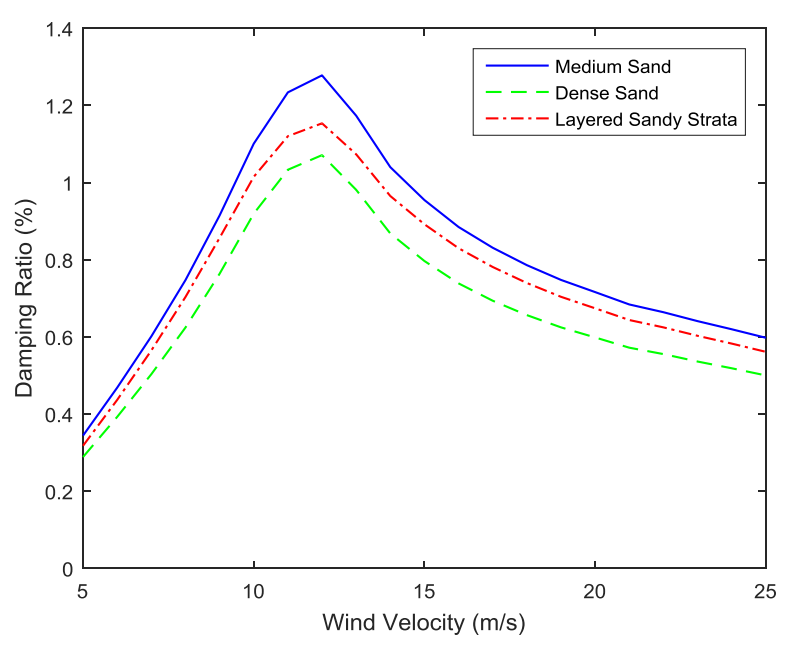

(d)

Figure 9 Soil damping in terms of wind speed for various soil profiles and p-y curves. (a) shows the clay profiles using static p-y curves; (b) shows the clay profiles using 
cyclic p-y curves; (c) shows the sand profiles using static p-y curves; (d) shows the sand profiles using cyclic p-y curves.

The plots in Figure 9 all show a similar behaviour. Soil damping ratios increase from $0.2 \%-0.3 \%$ at low wind speeds up to around $1.3 \%$ at the rated wind speed $12 \mathrm{~m} / \mathrm{s}$. Thereafter it decreases back to $0.3 \%-0.6 \%$. This range is in agreement with the published results reported in section 2.5 and our results confirm that soil damping is quite variable and can become quite significant for some tower amplitudes.

To understand these results better, Figure 10 (a) shows the average thrust for varying wind speed and Figure 10 (b) shows the modal stiffnesses for the clay soils obtained with static p-y curves. The behaviour for sands is very similar so the corresponding graph is not shown.

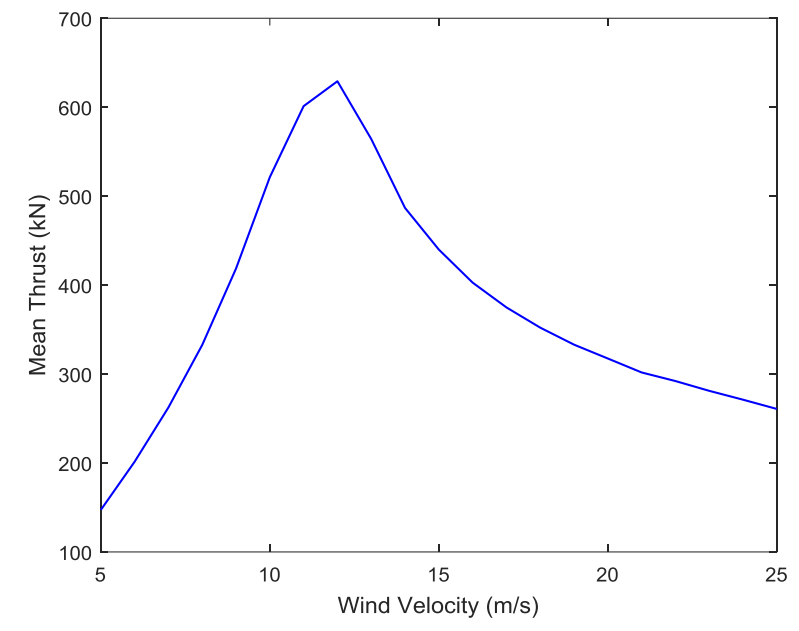

(a)

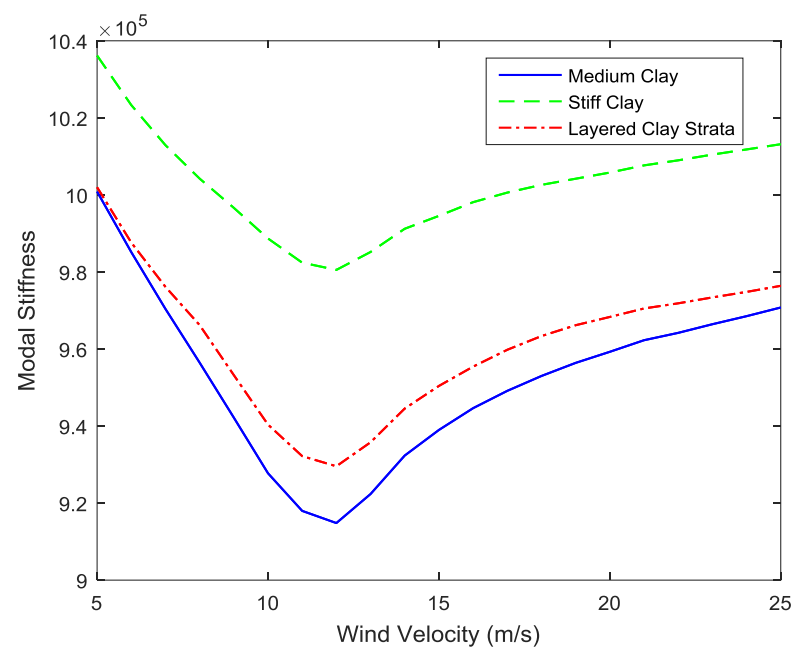

(b)

Figure 10 (a) Wind speed-thrust at tower top relationship (b) Modal stiffness (no physical unit) in terms of wind speed using static p-y curve soil model

Figure 10 (a) shows that up to the rated wind speed, the thrust increases. Then the control system starts feathering the blades so that the overall thrust decreases despite increasing wind speeds. It can be seen that the shape of the damping curves in Figure 9 follow closely that of the thrust.

This phenomenon is a consequence of two concomitant mechanisms (1) the non-linear softening of the soil as described by p-y curves (Figure 8) and (2) the increase in soil material damping with increasing strain described by Figure 7. When the deflection caused by the wind speed increases beyond some critical values, (the abrupt change in 
slope in the p-y curves), the soil stiffness decreases sharply, which reduces the stiffness of the whole system. This stiffness reduction leads to an increase in the soil damping contribution. The drop in stiffness in Figure 10(b) occurs when the wind speed is around the rated wind speed. This is when the largest rotor thrust and thus the largest displacement amplitude is experienced. According to Equation (30), the increased displacement magnitude also results in increased soil material damping ratio. As the constant of each soil dashpot increases the overall soil damping is further increased.

It should be noted that the above observations are based on the assumption that the soil behaviour is well described by p-y curves. Some soils actually stiffen due to cyclic loading (ratchetting behaviour) and in those cases the dependence of soil damping on the wind speed may be very different. This should be the subject of further study.

\section{Conclusion}

Vibration damping in offshore wind turbines is a critical parameter to predict reliably the dynamic response and fatigue life of the system. Although much research work has been done to characterise these sources of dissipation in OWTs, published values vary widely and a lot of uncertainty remain as to what should be used at the design stage, when actual measurements are not available. Following a detailed literature survey, this paper proposed a series of simple physics-based models that allowed the quantification of aerodynamic, hydrodynamic and soil damping separately for monopile supported OWTs. The dissipation models were implemented around a bespoke finite element beam model of the system based on the 5MW NREL offshore wind turbine. The damping results in this study are for the first fore-aft or side-side mode, which dominates the OWT dynamic response. These damping values can be easily implemented in a FE model at the design stage either specifying the damping ratio for the first mode or by inputting Rayleigh damping coefficients so that the first mode damping reaches the desired level.

Results showed that aerodynamic damping is in the range of $5-7 \%$ in the FA direction and $0.2-1.6 \%$ in the SS direction depending on the wind speed. Preliminary findings also pointed to the importance of the tilt angle and coupling between FA and SS directions although more work is needed to characterise this better. 
The viscous and radiation component of hydrodynamics damping were modelled from first principle. Results showed that for the current size of offshore wind turbines, hydrodynamic damping is much lower than what is usually quoted in the literature and for all practical purposes could be safely ignored.

Soil damping was modelled in the form of dissipative springs distributed along the embedded length of the pile. The properties of these springs are based on relevant p-y curves, which are nonlinear and as such their overall dissipative effect depends on the amplitude of vibration. Soil damping was calculated for different types of soils and incoming wind speeds. Results showed that $\zeta_{\text {soil }}$ ranges from $0.2 \%$ to $1.3 \%$ with a strong dependence on the stiffness of the system. As the thrust increases, the springs weaken so the amplitude increases relatively more. This increases the material damping and decrease the overall stiffness of the stiffness, thereby increasing the damping ratio. This effect has not previously been studied systematically.

Figure 11 combines the results from the various models by adding up the contribution from various damping sources in the fore-aft direction. $\zeta_{\text {struct }}=1 \%$ was used. The similarity between the fore-aft damping curves in Figure 11 and Figure 4 shows that the overall damping is completely dominated by the aerodynamic component. It increases sharply from $6 \%$ to $9 \%$ up to the rated wind speed and then decreases slowly down to $8 \%$.

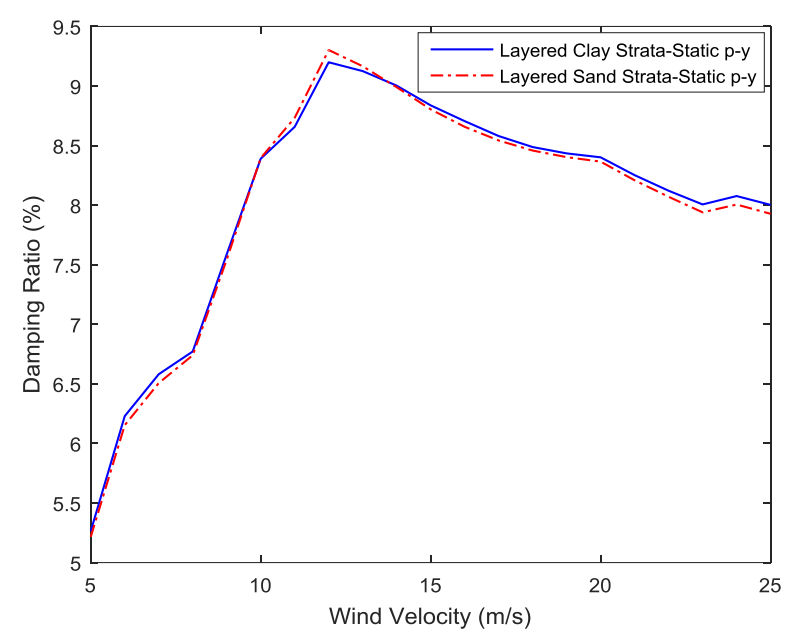

Figure 11 Overall FA damping ratio in a 5MW offshore wind turbine in terms of wind speed for two representative soil profiles. 
Outside the operative range of the turbine, the aerodynamic damping drops dramatically so that the overall damping is dominated by the structural and soil components. Therefore, a reasonable range for the total damping in a non-operating turbine could be between $1 \%$ and $1.5 \%$.

If the purpose of calculating the dynamic response of the system to design a currently non-existing turbine, it is important to get the damping values in the correct range always bearing in mind that lower damping values will lead to safe but potentially overconservative designs. By testing published values against simple but robust models, this paper can hopefully contribute to more optimal OWT designs.

From this study, two issues emerge as particularly deserving of further investigation: 1) there is still no data available for the structural damping in large OWTs even though this contribution is probably not insignificant; (2) Given the importance of the aerodynamic damping component, there is comparatively little reliable experimental data available to test numerical predictions. In fact, what seems to be needed first is a robust experimental methodology to measure and characterise this damping source properly. 


\section{References}

[1] European Wind Energy Association, "The European offshore wind industry key trends and statistics 2013," 2014.

[2] C. Fontana, W. Carswell, S. R. Arwade, D. J. DeGroot, and A. T. Myers, "Sensitivity of the Dynamic Response of Monopile-Supported Offshore Wind Turbines to Structural and Foundation Damping," Wind Eng., vol. 39, no. 6, pp. 609-628, 2015.

[3] J. M. Jonkman and M. L. J. Buhl, "FAST user's guide - updated august 2005," Technical Report No.NREL/TP-500-38230. National Renewable Energy Laboratory (NREL), Golden, CO, Oct. 2005.

[4] Wind Europe, "The European offshore wind industry - Key trends and statistics 2016," 2017.

[5] Windenergie GL, "Overall damping for piled offshore support structures," in Guideline for the Certification of Offshore Wind Turbines, 2005.

[6] W. G. Versteijlen, A. V. Metrikine, J. S. Hoving, E. H. Smid, and W. E. De Vries, "Estimation of the vibration decrement of an offshore wind turbine support structure caused by its interaction with soil," in Proceedings of the EWEA Offshore 2011 Conference, 2011.

[7] M. Damgaard and J. K. F. Andersen, "Natural frequency and damping estimation of an offshore wind turbine structure," in The Twenty-second International Offshore and Polar Engineering Conference, 2012.

[8] M. Damgaard, J. K. F. Andersen, L. B. Ibsen, and L. V. Andersen, "Timevarying dynamic properties of offshore wind turbines evaluated by modal testing," in Proceedings of the 18th International Conference on Soil Mechanics and Geotechnical Engineering, 2013, pp. 2343-2346.

[9] M. Damgaard, L. B. Ibsen, L. V. Andersen, and J. K. F. Andersen, "Cross-wind modal properties of offshore wind turbines identified by full scale testing," $J$. Wind Eng. Ind. Aerodyn., vol. 116, pp. 94-108, 2013.

[10] N. J. Tarp-Johansen, L. Andersen, E. D. Christensen, C. Mørch, B. Kallesøe, and S. Frandsen, "Comparing sources of damping of cross-wind motion," in The European Wind Energy Conference, The European Wind Energy Association, 2009. 
[11] R. Shirzadeh, C. Devriendt, M. A. Bidakhvidi, and P. Guillaume, "Experimental and computational damping estimation of an offshore wind turbine on a monopile foundation," J. Wind Eng. Ind. Aerodyn., vol. 120, no. SEPTEMBER, pp. 96-106, 2013.

[12] C. Devriendt, M. El-Kafafy, G. De Sitter, and P. Guillaume, "Damping estimation of offshore wind turbines on a monopile foundation using state-ofthe-art operational modal analysis techniques," Proc. Int. Conf. Noise Vib. Eng. ISMA 2012, pp. 2647-2662, 2012.

[13] C. Devriendt, P. J. Jordaens, G. De Sitter, and P. Guillaume, "Damping estimation of an offshore wind turbine on a monopile foundation," Proc. Int. Conf. Noise Vib. Eng. ISMA 2012, pp. 4411-4419, 2012.

[14] R. Shirzadeh, W. Weijtjens, P. Guillaume, and C. Devriendt, "The dynamics of an offshore wind turbine in parked conditions: A comparison between simulations and measurements," Wind Energy, vol. 18, no. 10, pp. 1685-1702, 2014.

[15] C. Devriendt, P. J. Jordaens, Y. Van Ingelgem, G. De Sitter, and P. Guillaume, "Monitoring of resonant frequencies and damping values of an offshore wind turbine," IET Renew. Power Gener., vol. 8, no. 4, pp. 433-441, 2014.

[16] C. Koukoura, A. Natarajan, and A. Vesth, "Identification of support structure damping of a full scale offshore wind turbine in normal operation," Renew. Energy, vol. 81, pp. 882-895, 2015.

[17] M. H. Hansen, K. Thomsen, P. Fuglsang, and T. Knudsen, "Two methods for estimating aeroelastic damping of operational wind turbine modes from experiments," no. January, pp. 179-191, 2006.

[18] M. O. L. Hansen, Aerodynamics of wind turbines. $2^{\text {nd }}$ Edition, Earthscan, 2008.

[19] V. Valamanesh and A. T. Myers, "Aerodynamic damping and seismic response of horizontal axis wind turbine towers," J. Struct. Eng., vol. 140, no. 11, pp. 19, 2014.

[20] M. Ozbek, F. Meng, and D. J. Rixen, "Challenges in testing and monitoring the in-operation vibration characteristics of wind turbines," Mech. Syst. Signal Process., vol. 41, no. 1-2, pp. 649-666, 2013.

[21] D. J. C. Salzmann and J. Van Der Tempel, “Aerodynamic damping in the design of support structures for offshore wind turbines," Proc Copenhagen Offshore Conf, p. 9 pp, 2005. 
[22] S. Schafhirt and M. Muskulus, "Decoupled simulations of offshore wind turbines with reduced rotor loads and aerodynamic damping," Wind Energy Sci., vol. 3, no. 1 , p. $25,2018$.

[23] M. Ozbek and D. J. Rixen, "Operational modal analysis of a 2.5 MW wind turbine using optical measurement techniques and strain gauges," Wind Energy, vol. 17, pp. 367-381, 2013.

[24] O. Faltinsen, Sea loads on ships and offshore structures. Cambridge University Press, 1993.

[25] M. F. Cook and J. K. Vandiver, "Measured and predicted dynamic response of a single pile platform to random wave excitation," in Proceedings of 14th Annual Offshore Technology Conference in Texas, 1982.

[26] W. Carswell, J. Johansson, F. Løvholt, S. R. Arwade, C. Madshus, D. J. DeGroot, and A. T. Myers, "Foundation damping and the dynamics of offshore wind turbine monopiles," Renew. Energy, vol. 80, pp. 724-736, 2015.

[27] M. Novak, "Dynamic stiffness and damping of piles," Canadian Geotechnical Journal, vol. 11, no. 4. pp. 574-598, 1974.

[28] G. Gazetas and R. Dobry, "Horizontal response of piles in layered soils," $J$. Geotech. Eng., vol. 110, no. 1, pp. 20-40, 1984.

[29] M. H. El Naggar and K. J. Bentley, "Dynamic analysis for laterally loaded piles and dynamic p-y curves," Can. Geotech. J., vol. 37, no. 6, pp. 1166-1183, 2000.

[30] H. B. Seed and Idriss I. M., "Soil moduli and damping factors for dynamic response analyses," Report NO. EERC 70-10. California Univ Berkely Earthquake Engineering Research Centre, 1970.

[31] M. B. Darendeli, "Development of a new family of normalized modulus reduction and material damping curves," $\mathrm{PhD}$ Dissertation, The University of Texas at Austin, 2001.

[32] EN 1991-1-4, "Eurocode 1: Actions on structures -Part 1-4: General actions Wind actions," Eur. Comm. Stand., vol. 4, no. 2005, pp. 1-148, 2005.

[33] N. D. P. Barltrop and A. J. Adams, Dynamics of fixed marine structures. Butterworth-Heinemann, 1991.

[34] P. Schaumann and M. Seidel, "Eigenschwingverhalten von windenergieanlagen-berechnungen und messungen," in Tagungsband DEWEK, pp. 35-38, 2000.

[35] R. Rezaei, P. Fromme, and P. Duffour, "Fatigue life sensitivity of monopile- 
supported offshore wind turbines to damping," Renew. Energy, vol. 123, pp. 450-459, 2018.

[36] J. M. Jonkman, S. Butterfield, W. Musial, and G. Scott, "Definition of a 5-MW reference wind turbine for offshore system development," Technical Report NREL/TP-500-38060 February 2009, National Renewable Energy Laboratory (NREL), Golden, CO., 2009.

[37] H. P. Gavin, "Numerical integration in structural dynamics," Department of Civil \& Environmental Engineering Duke University. Lecture Notes, Department of Civil \& Environmental Engineering Duke University. 2016.

[38] C. H. Hodges, J. Power, and J. Woodhouse, "The use of the sonogram in structural acoustics and an application to the vibrations of cylindrical shells," $J$. Sound Vib., vol. 101, no. 2, pp. 203-218, 1985.

[39] M. O. L. Hansen, J. N. Sørensen, S. Voutsinas, and H. A. Madsen, "State of the art in wind turbine aerodynamics and aeroelasticity," Prog. Aerosp. Sci., vol. 42, no. 4, pp. 285-330, 2006.

[40] T. Sarpkaya, Wave forces on offshore structures. Cambridge University Press, 2010.

[41] L. Johanning, P. W. Bearman, and J. M. R. Graham, "Hydrodynamic damping of a large scale piercing circular in planar oscillatory motion," J. Fluids Struct., vol. 16, no. 15, pp. 891-908, 2001.

[42] B. Petersen, M. Pollack, B. Connell, D. Greeley, D. Davis, C. Slavik, and B. Goldman, "Evaluate the effect of turbine period of vibration requirements on structural design parameters: technical report of findings," 2010.

[43] R. Salmon, "Introduction to Ocean Dynamics," Lecture Notes, University of California, San Diego, 2000.

[44] J. Van Der Tempel, "Design of Support Structures for Offshore Wind Turbines," PhD Dissertation, Delft University of Technology, 2006.

[45] G. L. Kuiper, A. V. Metrikine, and J. A. Battjes, "A new time-domain drag description and its influence on the dynamic behaviour of a cantilever pipe conveying fluid," J. Fluids Struct., vol. 23, no. 3, pp. 429-445, 2007.

[46] J. R. Chaplin, "Hydrodynamic damping of a cylinder at $\beta \approx 10^{6}$," J. Fluids Struct., vol. 0, pp. 1101-1117, 2000.

[47] M. F. Cook, "Damping estimation, response prediction and fatigue calculation of an operational single pile platform," Master Dissertation, Massachusetts 
Institute of Technology, 1978.

[48] C. Petrauskas, "Hydrodynamic damping and "added mass" for flexible offshore platforms." Technical Paper No. 76-18, California Univ Berkely Hydraulic Engineering Lab, 1976.

[49] P. Jamieson, Innovation in wind turbine design. John Wiley \& Sons, 2011.

[50] E. Van Buren and M. Muskulus, "Improving pile foundation models for use in bottom-fixed offshore wind turbine applications," Energy Procedia, vol. 24, no. 1876, pp. 363-370, 2012.

[51] N. Subramanian, Steel Structures - Design and Practice. Oxford University Press, 2011.

[52] DNV, Design of Offshore Wind Turbine Structures, DNV-OS-J101, 2014.

[53] API, Recommended Practice for Planning, Designing and Constructing Fixed Offshore Platforms - Working Stress Design, API Recommended Practice 2AWSD, 2000.

[54] L. Reese, W. Van Impe, and R. Holtz, Single piles and pile groups under lateral loading. CRC Press, 2010. 


\section{Appendix A}

\section{Derivation for side-side aerodynamic damping}
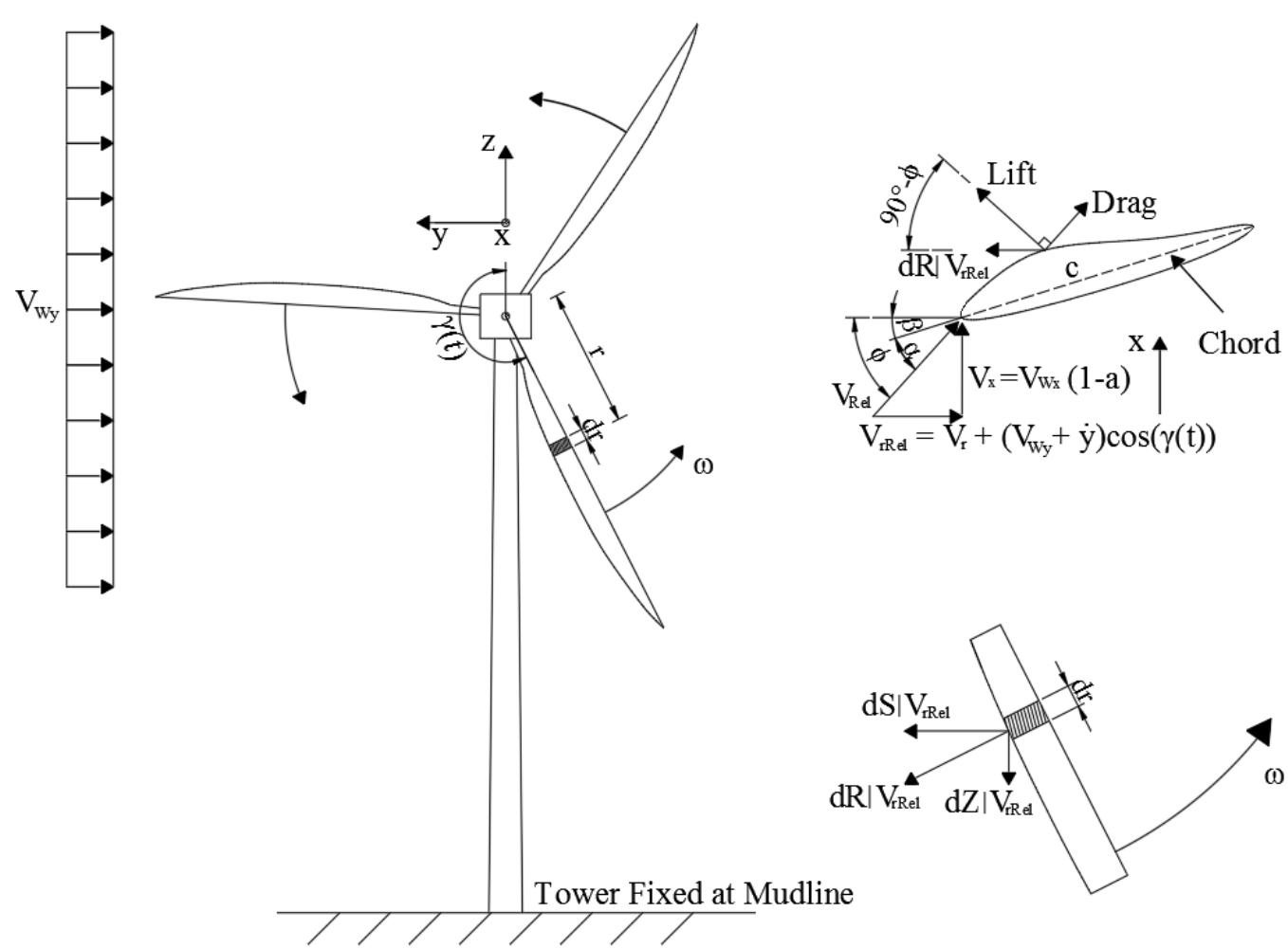

Figure A1 Blade element forces for side-side vibration

For the side-side direction a similar approach can be followed. Calling $V_{r}$ the speed component in the tangential direction, BEM theory gives $V_{r}=\omega r\left(1+a^{\prime}\right)+$ $V_{W y} \cos \left(\gamma_{i}(t)\right)$ [18] where $V_{W y}$ is the inflow wind speed in side-side direction and $\gamma_{i}(t)$ is the azimuth angle of the blade which is a function of time $t$ when the turbine is in operation. The force in the $y$ direction felt by an element at distance $r$ along the ith blade is

$$
\left.d S\right|_{V_{r}}=\frac{1}{2} \rho_{a} \frac{V_{r}^{2}}{\cos ^{2} \phi} c C_{t} \cos \left(\gamma_{i}(t)\right) d r
$$

where $C_{t}=C_{l} \sin \phi-C_{d} \cos \phi$ and $\gamma_{i}(t)$ is the azimuth angle of the blade which is a function of time $t$ when the turbine is in operation. It is noted that here the elemental force in y direction is assumed to be a function of $V_{r}$ and $\phi$. If there is a small change in $V_{r}$ due to the overall tower top motion, the relative speed of the element in tangential direction $V_{\text {rRel }}$ becomes: 


$$
V_{\text {rRel }}=V_{r}+\left(V_{W y}+\dot{y}\right) \cos \left(\gamma_{i}(t)\right) .
$$

In the following derivation we can always assume that $V_{W y}=0$ since the turbine can be controlled and oriented so as to face the wind. Again a first order Taylor's expansion of the side-side force gives the expression of $\left.d S\right|_{V_{r R e l}}$ which is the tangential force felt by the blade element under a relative speed of $V_{\text {rRel }}$ :

$$
\begin{gathered}
\left.d S\right|_{V_{r R e l}}=\left.d S\right|_{V_{r}}+\left.d S^{\prime}\right|_{V_{r}} \cdot\left(V_{r R e l}-V_{r}\right) \\
=\left.d S\right|_{V_{r}}+\frac{d(d S)}{d V_{r}} \dot{y} \cos \left(\gamma_{i}(t)\right) .
\end{gathered}
$$

Assuming that $d S$ is a function of $V_{r}$ and $\phi$, and $\phi$ is a function of $V_{r}, \frac{d(d S)}{d V_{r}}$ can be calculated as:

$$
\frac{d(d S)}{d V_{r}}=\frac{\partial(d S)}{\partial V_{r}}+\frac{\partial(d S)}{\partial \phi} \frac{d \phi}{d V_{r}}
$$

$\frac{\partial(d S)}{\partial V_{r}}$ and $\frac{\partial(d S)}{\partial \phi}$ can be obtained by differentiating Equation ( A. 1):

$$
\frac{\partial(d S)}{\partial V_{r}}=\rho_{a} \frac{V_{r}}{\cos ^{2} \phi} c C_{t} \cos \left(\gamma_{i}(t)\right) d r,
$$

$$
\begin{aligned}
& \frac{\partial(d S)}{\partial \phi} \\
& =\frac{1}{2} \rho_{a} V_{r}^{2} \frac{\frac{d C_{t}}{d \phi} \cos ^{2} \phi+2 \cos \phi \sin \phi C_{t}}{\cos ^{4} \phi} \cos \left(\gamma_{i}(t)\right) d r .
\end{aligned}
$$

To obtain an expression for $\frac{d \phi}{V_{r}}$, the following two equations can be used:

$$
\begin{gathered}
\cot \phi=\frac{V_{r}}{V_{W x}(1-a)}, \\
a=\frac{1}{\frac{4 \sin ^{2} \phi}{\sigma C_{n}}+1} .
\end{gathered}
$$

Differentiating Equation (A. 7) with respect to $V_{r}$ and Equation (A. 8) with respect to $\phi$, resulting in

$$
\frac{d \phi}{d V_{r}}=\frac{1}{V_{W x}\left(\frac{1-a}{\sin ^{2} \phi}-\frac{d a}{d \phi} \cot \phi\right)}
$$

and 


$$
\frac{d a}{d \phi}=\frac{-4\left(\sin 2 \phi C_{n}-\frac{d C_{n}}{d \phi} \sin ^{2} \phi\right)}{\sigma C_{n}^{2}\left(\frac{4 \sin ^{2} \phi}{\sigma C_{n}}+1\right)^{2}}
$$

Equations ( A. 5), ( A. 6) and ( A. 9) can be substituted into Equation ( A. 4), and the resultant $\frac{d(d S)}{d V_{r}}$ can be used to obtain $\left.d S\right|_{V_{r R e l}}$ in Equation ( A. 3) which is the sideside aerodynamic force subjected by the rotor element. For simplicity, let

$$
\begin{gathered}
A^{\prime} \cos \left(\gamma_{i}(t)\right) \cdot d r=\frac{\partial(d S)}{\partial V_{r}}, \\
B^{\prime} \cos \left(\gamma_{i}(t)\right) \cdot d r=\frac{\partial(d S)}{\partial \phi} \frac{d \phi}{d V_{r}} .
\end{gathered}
$$

So, the side-side aerodynamic force for one element is

$$
\left.d S\right|_{V_{r R e l}}=\left.d S\right|_{V_{r}}+\left(A^{\prime}+B^{\prime}\right) d r \cdot \dot{y} \cos ^{2}\left(\gamma_{i}(t)\right) .
$$

The side-side aerodynamic force felt by the ith blade should be

$$
\begin{aligned}
\left.F\right|_{y_{i}} & =\left.\int_{0}^{R} d S\right|_{V_{r R e l}} \\
& =\left.\int_{0}^{R} d S\right|_{V_{r}}+\int_{0}^{R}\left(A^{\prime}+B^{\prime}\right) d r \cdot \dot{y} \cos ^{2}\left(\gamma_{i}(t)\right) .
\end{aligned}
$$

Following a similar procedure to that outlined in the fore-aft case, the total side-side aerodynamic force felt by the rotor can be expressed as:

$$
F_{y}=\left.\sum_{i=1}^{N_{b}} \int_{0}^{R} d S\right|_{V_{r}}+\sum_{i=1}^{N_{b}} \int_{0}^{R}\left(A^{\prime}+B^{\prime}\right) d r \cdot \dot{y} \cos ^{2}\left(\gamma_{i}(t)\right) .
$$

For a symmetric three-blade rotor on steady condition without vibration and inflow wind in side-side direction, the total force on side-side direction, $\left.\sum_{i=1}^{N_{b}} \int_{0}^{R} d S\right|_{V_{\text {rRel }}}$ is zero, so $d F_{y}$ can be simplified to

$$
F_{y}=\frac{N_{b}}{2} \int_{0}^{R}\left(A^{\prime}+B^{\prime}\right) d r \cdot \dot{y}
$$

since $\left.\sum_{i=1}^{N_{b}} \int_{0}^{R} d S\right|_{V_{r}}=0$ and $\sum_{i=1}^{N_{b}} \cos ^{2}\left(\gamma_{i}(t)\right)=\frac{N_{b}}{2}$. It should be noted that the term $A^{\prime}+B^{\prime}$ is negative. 


\section{Appendix B}

\section{Derivation for radiation damping coefficient for wave-cylinder interaction}

The radiation force applied on the cylinder highly depends on its oscillating frequency $\sigma$, and the well-known linear dispersion relation is important for the radiation problem. The linear dispersion relation can be written as

$$
\sigma^{2}=g k \cdot \tanh (k h)=-g k_{n} \cdot \tan \left(k_{n} h\right), \quad n=1,2,3, \ldots
$$

where $g$ is the gravitational acceleration, $k$ is the wavenumber and $h$ is the depth of the water. Assuming the distributed hydrodynamic force over the height of tower under water is $f_{\text {Rad }}(z, t), \bar{f}(t)$ in Equation (5) excluding other damping terms can be written as

$$
\bar{f}(t)=\int_{-h}^{0} f_{R a d}(z, t) \boldsymbol{\Phi}(z) d z
$$

According to Charles Petrauskas [48] who solves the PDEs for velocity potentials, $f_{\text {Rad }}(z, t)$ can be expressed by

$$
f_{R a d}(z, t)=-\rho_{w} \pi a \sigma h G_{0}(k h) P_{1}(k a) \cosh (k(z+h)) \cdot \dot{\alpha}(t),
$$

where

$$
\begin{gathered}
G_{0}(k h)=\frac{2 \int_{-h}^{0} \cosh (k(z+h)) \Phi(z) d z}{h(\sinh (k h) \cosh (k h)+k h)}, \\
P_{1}(k a)=\frac{2}{\pi k a\left(J_{1}^{\prime}(k a)^{2}+Y_{1}^{\prime}(k a)^{2}\right)} .
\end{gathered}
$$

$J_{1}^{\prime}(k a)$ and $Y_{1}^{\prime}(k a)$ are the derivatives of the Bessel functions of the first kind and second kind respectively. Denoting

$$
C_{R a d}=\rho_{w} \pi a \sigma h G_{0}(k h) P_{1}(k a) \cosh (k(z+h)) \boldsymbol{\Phi}(z),
$$

$\bar{f}(t)$ can be written as

$$
\bar{f}(t)=-\int_{-h}^{0} C_{R a d} d z \cdot \dot{\alpha}(t) .
$$

